\title{
'Gritos de cerveza, cerveza': el rol de la música y de los DJs en el control del desorden en los clubes nocturnos
}

\section{'Lager, lager shouting': The role of music and DJs in nightclub disorder control}

\author{
| ALASDAIR J. M. FORSYTH
}

Scottish Centre for Crime and Criminal Justice Research

Glasgow Caledonian University. United Kingdom, Reino Unido.

Enviar correspondencia a:

Alasdair J M Forsyth, Department of Psychology, Glasgow

Caledonian University, Cowcaddens Road, Glasgow G4 OBA, UK.

Tel. 0141-331-8301. Fax: 0141-331-3636.

E-mail: Alasdair.forsyth@gcal.ac.uk.

\section{RESUMEN}

Objetivos: Este articulo explora cómo la música y los géneros musicales pueden regir la experiencia de la vida nocturna y, en especial, de qué forma la política musical de un club nocturno puede tener un impacto en la clientela, los hábitos saludables, las ventas en la barra del bar y los niveles de desorden.

Materiales y métodos: Se llevaron a cabo observaciones participantes en una muestra de ocho clubes nocturnos del centro de Glasgow, Escocia. Resultados: Se encontró que la politica musical ejercia una influencia tanto en los clientes de los clubes como en sus comportamientos, por ejemplo, en relación a los niveles de consumo de alcohol y otras drogas, a la actividad sexual y a la violencia dentro de los locales. Es más, dentro de cada local, también se examinó la política musical como herramienta de control de la multitud, y la utilización de dicho entretenimiento, por ejemplo, en la venta de alcohol, en la vigilancia laxa y en la gestión temporal de los movimientos de los clientes. Esta investigación reconoce el papel central del DJ en la implementación de estos controles.

Conclusiones: Está claro que la música constituye un componente central en la economía nocturna. A pesar de ello, hasta ahora, la música sólo ha jugado un papel secundario, si la comparamos con el sexo y las drogas, dentro de las investigaciones del Club Health. A partir de esta investigación, recomendamos que la política musical sea incorporada dentro de los programas de formación en dispensación responsable. recibido: diciembre 2008 aceptado: junio 2009

\section{ABSTRACT}

Objective: This paper explores how music and music genre can govern the nightlife experience, specifically how a nightclub's music policy can impact on clientele, health behaviours, bar sales and levels of disorder.

Material and method: Participant observations were conducted in sample of eight city centre nightclubs in Glasgow, Scotland.

Results: Music policy was found to influence nightclubs' clientele and their behaviours, for example in relation to differences in levels of alcohol or illegal drug use, sexual activity and violence between venues. Further, within individual venues, music policy was also observed operating as a crowd control tool, with for example such entertainment being used in alcohol marketing, in 'soft policing' and in the temporal management of patrons' movements. This research acknowledges the centrality of the DJ in implementing these controls.

Conclusions: It is clear that music is a central component of the Nighttime Economy. Despite this, to date music has tended to play 'second fiddle' to sex and drugs within Club Health research. From this research it is recommended that music policy be incorporated into responsible bar server training packages.

Key words: nightclubs, music, alcohol, drugs, violence 


\section{INTRODUCCIÓN}

$\mathrm{E}$ la canción 'Sing for the moment' (2002) el rapero americano Eminem plantea "dicen que la música puede alterar los estados de humor y hablarte ¿pero puede también cargar una pistola y dispararla?". Este argumento es manifiesto del poder de la música para influenciar el comportamiento humano. Sube el volumen; añade alcohol y/o otras drogas, y entonces la influencia que la música ejerce sobre las actividades nocturnas devendrá más evidente. En algunas circunstancias, la música puede hacer que los individuos más plácidos 'se levanten y bailen', mientras que en otras circunstancias puede ser un detonante para que se embarquen en comportamientos desordenados. Pero a pesar de esta asociación obvia, hasta la fecha, las investigaciones sobre la vida nocturna han tendido a pasar por alto la música, concentrándose en las otras dos fuerzas con mayor potencial dentro de la dicha economía, a saber drogas/alcohol y competición sexual'.

En su himno 'Sexo y drogas y rock n' roll' (1977) el ya fallecido cantante punk-rock lan Dury argüía que estos tres elementos "son todo lo que mi cerebro y mi cuerpo necesitan". A pesar de las implicaciones para la salud de este sentimiento, llama la atención que se haya prestado tan poca atención académica al papel que juega la música en la economía nocturna, incluyendo los trabajos del Club Health (si exceptuamos las sobresalientes investigaciones sobre los efectos del volumen y la pérdida de oído ${ }^{2,3}$ ). Dado que uno puede solazarse mediante actuaciones sexuales activadas por las drogas dentro de la seguridad y el confort de la propia casa ¿qué otras razones hay para aventurarse a la economía nocturna, con todos los riesgos (violencia, heridas accidentales, arrestos, gastos, consumo de tiempo, etc. ${ }^{4}$ ) que dicha aventura comporta, si no fuera encontrar el entretenimiento? En términos de la experiencia de los clubes nocturnos, el entretenimiento ofrecido es la música de baile, en un entorno social/de grupo.

Sorprendentemente, parece que hay más investigaciones académicas dirigidas a examinar el papel de la música en la economía diurna. Quizás el mejor ejemplo sea el trabajo de investigación de Tia DeNora sobre la 'música en la vida cotidiana'5. DeNora detalla cómo la música funciona como la banda sonora de nuestras vidas y para, como para Eminem (ver más arriba), alterar nuestro ánimo. Por consiguiente, los comercios al detall de la economía diurna utilizan la música para atraer a los consumidores, influenciar sus comportamientos de compra y demarcar los recorridos principales $^{6}$. DeNora también describe como el oyente/consumidor puede utilizar la música para influenciar deliberadamente sus propios estados de ánimo, en una especie de 'auto-programación', y que la gente parece saber qué tipo de música 'necesita' en una situación determinada (esto es, la música tiene propiedades parecidas a las drogas tales como la automedicación, la estimulación o la adicción). Por ejemplo, resulta de particular interés la convicción de DeNora de que la música puede emplearse para 'descargar' (es decir, para desahogarse o evitar una agresión).

\section{INTRODUCTION}

$n$ the song 'Sing for the Moment' (2002) American rapper Eminem states that "They say music can alter moods and talk to you but can it load a gun up and cock it too?" This argument is testament to the power of music to influence human behaviour. Turn up the volume; add alcohol and I or other drugs, then the influence that music has over nightlife activities can become even more apparent. In some circumstances, music can make the most placid individuals 'get up and dance', while in other circumstances, it can be a trigger for them to engage in disorderly behaviour. Yet despite this obvious association, to date nightlife research has tended to overlook music, instead concentrating on the other two driving forces within the Night-time Economy, namely drugs / alcohol and sexual competition 1 .

In his anthem 'Sex and drugs and rock n' roll' (1977) the late English punk rock singer, lan Dury argued that these three elements "are all my brain and body need". The health implications of such sentiment notwithstanding, it is striking that so little academic attention has been paid to the role of music in the Night-time Economy, including Club Health (with the noteworthy exception of research into the effects of volume and hearing loss ${ }^{2,3}$ ). Given that one can engage in drug-fuelled sexual behaviours in the safety and comfort of one's own home, what other reasons can there be for venturing out into the Night-time Economy, with all the risks (e.g. violence, accidental injury, arrest, expense, time consumption, etc. ${ }^{4}$ ) that such a venture entails, were it not to find entertainment? In terms of the nightclub experience the entertainment on offer is dancing to music, in a social / group setting.

Surprisingly there would appear to have been more academic research conducted about the role of music in the Day-time Economy. Perhaps the best example of this work is Tia DeNora's research into 'music in everyday life'5. DeNora details how music can act as the soundtrack to our lives and, like Eminem (see above), alter our moods. Accordingly retailers in the Day-time Economy are seen to use music to attract consumers, influence their purchasing behaviour and demarcate the High Street ${ }^{6}$. DeNora also describes how the listener / consumer can use music purposively to influence their own moods, in a sort of 'self-programming', in that people seem to know what kind of music they 'need' in a given situation (i.e. music has drug-like properties, such as self-medication, stimulation or addiction). For example, of particular interest here is DeNora's belief that music can be used for 'venting' (i.e. letting off steam or acting out aggression).

"I just go to my room, slam the door, play my music and just sort of feel mad for a couple more minutes... When I turn the music up real loud it fills my room, it's like I can't hear anything outside my room and just me really mad" 7 
"Voy a mi habitación, doy un portazo, pongo mi música y me siento furioso durante un par de minutos más... Cuando pongo la música realmente fuerte llena toda la habitación, es como si no pudiera oír nada de lo que ocurre fuera de mi habitación y estoy únicamente yo completamente loco" 7

Una investigación en curso de North (detallada por Fegaon y Carmichael ${ }^{8}$ y Michaels ${ }^{9}$ ) indica que los gustos musicales son un reflejo de la personalidad. En este estudio internacional, North encontró que la amabilidad (es decir, la agresividad) de un individuo puede predecirse de esta manera. Sin embargo como los fans 'amables' preferían géneros musicales que incluian metal, disco, grandes éxitos, reggae y soul, mientras que los denominados 'no amables' incluian fans del rock n' roll, punk, indie y dance, las implicaciones en los desórdenes nocturnos no son todavía claros.

Previamente, North, con Hargreaves ${ }^{10}$, había encontrado que la música podía influenciar los comportamientos de compra de alcohol, aunque, de nuevo, esta investigación se desarrolló en el contexto de la economía diurna, en supermercados. En concreto, los consumidores ingleses compraban más vino francés o alemán si, en los comercios donde se encontraban, la música ambiental estaba asociada estereotípicamente a ese país. En un estudio similar, Areni y $\mathrm{Kim}^{11}$ encontraron que poner música clásica en una tienda de vinos conducía a los consumidores a comprar vinos más caros que si se tocaban éxitos populares, aunque el número de botellas adquiridas no se vio afectado. La hipótesis era que la música clásica indicaba un establecimiento más 'exclusivo'.

En los EE.UU., la imagen positiva del alcohol en las letras de las canciones emitidas en las radios orientadas a los jóvenes ha sido identificada como un motivo de preocupación ${ }^{12}$, al tiempo que también levantaba preocupación la imagen positiva que daban de sus propios hábitos de bebida los DJs en programas diurnos de las radios generalistas en el Reino Unido ${ }^{13}$.

Lo que todos los estudios mencionados comparten es el punto de vista de que la música puede ejercer una influencia en los comportamientos de los individuos de la economía diurna, como la promoción del alcohol, que incluye sus creencias sobre el uso de sustancias (es decir, investigaciones que no toman en consideración el consumo actual del oyente/consumidor). Por el contrario, cuando se ha examinado el papel de la música en la economía nocturna, la investigación se ha centrado mayoritariamente en cómo afecta al índice de consumo de alcohol por grupos de oyentes. Por ejemplo, en EE.UU., Drews y otros colegas ${ }^{14}$ encontraron que la sola presencia de música en bares podía incrementar el consumo. En Francia, Gueguen et al. ${ }^{15}$, hallaron que la música más alta incrementaba de forma similar el consumo de alcohol, mientras que McElrea y Standing ${ }^{16}$ descubrieron que la música más rápida también incrementaba los índices de consumo de alcohol.

En contraste con estos hallazgos, otro estudio sobre bares, llevado a cabo por Bach y Schaefer ${ }^{17}$, encontró que el tempo de la música también afectaba a los índices de consumo. Sin embargo, en su estudio, la música country con un tempo más lento también incrementaba los indices de consumo de bebidas. Este hallazgo implicaba que era el efecto de ese tempo más lento sobre el ánimo de los oyentes (es decir, si les ponía tristes) lo que incrementaba el consumo de alcohol. Una inves-
Ongoing research by North (detailed by Fegaon \& Carmichael $^{8}$ and Michaels ${ }^{9}$ ) has indicated that musical tastes are a reflection of personality. In his international study, North has found that an individual's gentleness (read aggressiveness) could be predicted in this way. However as 'gentle' fans' preferred music genres included metal, disco, chart, reggae and soul, while those termed 'not gentle' included fans of rock $n$ ' roll, punk, indie and dance, the implications for nightlife disorder are unclear.

Previously, North, with Hargreaves ${ }^{10}$, had found that music could influence alcohol purchasing behaviour, though again this research was conducted in the Day-time Economy, in supermarkets. Specifically, that English consumers would purchase either more French or more German wine, if the shop concerned was playing background music stereotypically associated with that country. In a similar study Areni and $\mathrm{Kim}^{11}$ found that playing classical music in a wine store, led consumers to buy more expensive wine in comparison to playing chart music, thought the number of bottles purchased was unaffected. The hypothesis being that classical music indicated a more 'upscale' establishment.

In the USA, the positive portrayal of alcohol in music lyrics on youth-orientated radio has been identified as a cause for concern ${ }^{12}$, while more recently similar concerns have been raised about the effect on alcohol consumption by the positive portrayal of their own drinking habits by DJs on daytime UK-wide music radio ${ }^{13}$.

What all the above studies have in common is the view that music can influence an individual's behaviour in the Day-time Economy, including beliefs about substance use, such as alcohol promotion (i.e. research which involves no actual drinking by listener / consumer). In contrast, when the role of music has been examined in the Night-time Economy, this has mainly focused to how it affects groups of listeners' alcohol consumption rate. For example in the USA, Drews and colleagues ${ }^{14}$ found that the mere presence of music in bars could increase consumption. In France, Gueguen and Colleagues ${ }^{15}$ found that louder music similarly increased alcohol consumption, while McElrea and Standing $^{16}$ found that faster music also increased alcohol consumption rates.

In contrast to the above findings, another barroom study, by Bach and Schaefer ${ }^{17}$ also found that the tempo of music in bars affected consumption rates, however in their research country music with a slower tempo increased drinking rates. This finding implied that it was the effect that the slower forms of this genre had on listeners' mood (i.e. it made them sad) which increased alcohol consumption. Research conducted by Caldwell $\&$ Hibbert ${ }^{18}$ in restaurants in Glasgow, Scotland, (i.e. in the same city where the fieldwork for the present study was conducted) also found that slower music tempo could increase consump- 
tigación conducida por Caldwell y Hibbert ${ }^{18}$ en restaurantes de Glasgow, Escocia (la misma ciudad donde se ha llevado a cabo este estudio) encontró también que la música con un tempo más lento podía incrementar el consumo. En su estudio hallaron que la música más lenta animaba a los clientes a permanecer en el local durante un periodo más largo de tiempo, lo que influia tanto en el consumo de comida como de bebida, si bien lo hacía mediante el incremento del tiempo pasado en el establecimiento en vez de con un índice de consumo más rápido.

En Australia, Homel y Tomsen ${ }^{19}$ encontraron que la presencia de música, en especial de bandas en directo, proporcionaba distracción en entornos incómodos. Esto podría explicar algunos de los hallazgos de los estudios mencionados, en los que la atracción de la música puede invalidar otros efectos negativos, e influenciar a los clientes para permanecer durante un periodo más largo en los locales. Homel y Tomsen también apuntaron que la calidad de la musicalidad podía tener su impacto en los niveles de desorden.

El mencionado estudio de Homel y Tomsen se realizó durante una época en la que los DJs no eran elementos centrales en el funcionamiento de los locales con licencia, como es el caso en la economía nocturna globalizada de hoy en día. Un estudio más reciente de Hadfield ${ }^{20}$ señaló que los DJs podian jugar papeles similares a los descritos por Homel y Tomsen sobre las bandas. Describiendo a los DJs como "controladores guay", Hadfield (pág. 98-104) señaló cómo podía utilizarse a estos animadores, en vez de al personal de seguridad, para mantener a los clientes en un estado de ánimo donde la aparición de la violencia fuera poco probable. Por otro lado, Hadfield ${ }^{19}$ también mostró que los DJs deben mostrarse muy cautelosos con las sensibilidades locales, evitando por ejemplo canciones que puedan asociarse a equipos de fútbol concretos, ya que tocarlas podría ser un detonante para los desórdenes.

Investigaciones anteriores realizadas por el mismo autor en pubs de Glasgow, Escocia, hallaron tanto en las observaciones de campo como en las entrevistas al personal que la música era utilizada de forma estratégica por los operadores para reclutar clientes, retenerles y moldear sus comportamientos, en resumen, para incitarles a pasar más tiempo en el bar. Las consecuencias negativas de esta estrategia se duplican; primero, un incremento de las ventas en el bar significaba un aumento de probabilidades de desórdenes relacionados con el alcohol y, segundo, los incidentes con desórdenes violentos estaban a menudo centrados en la música, especialmente en torno al baile (en ausencia de pista de baile) y el karaoke. Sin embargo estas investigaciones previas se vieron limitadas en el hecho de que los pubs son también parte de la economía diurna (es decir, antes de medianoche), y que esos hábitos de consumo de sustancias es probable que sean menos variados y podría decirse que menos extremos que en los clubes (se observó que los clientes de los pubs de Glasgow se dirigían a los clubes cuando los primeros cerraban). También, el papel de la música es menos central en la función del pub. A diferencia de en los pubs, en los clubes las fuentes de música tales como el karaoke (y las máquinas de discos) es poco probable que sean una característica del entorno sónico; sin embargo estos locales (grandes) suelen tener pistas de baile designadas, actuaciones en directo, sistemas de sonido construidos tion. In their research slower music was found to encourage patrons to remain in the restaurant for a longer period of time, thus influencing both food and drink consumption, through increased time spent on the premises rather than via a faster drinking rate.

In Australia Homel and Tomsen ${ }^{19}$ found that the presence of music, particularly live bands, could provide distraction from uncomfortable surroundings. This may explain some of the findings of the above studies, in that the attraction music may over-ride other negative factors, influencing patrons to remain on premises for a longer time period. Homel and Tomsen ${ }^{19}$ also noted that the quality of musicianship could impact on levels of disorder.

The above study by Homel and Tomsen ${ }^{19}$ was conducted in an era when the DJ was less central to the functioning of licensed premises than is likely to be the case in today's globalising Night-time Economy ${ }^{4}$. More recent research by Hadfield ${ }^{20}$ found that DJs could perform similar roles to that of the live bands described by Homel and Tomsen ${ }^{19}$. Describing DJs as the "phat controllers", Hadfield (pp. 98-104) noted how these entertainers could be used instead of security staff to keep patrons in a mood where violence was unlikely to occur. On the other hand, Hadfield ${ }^{19}$ also noted that DJs had to be wary of local sensitivities, for example avoiding songs which may be linked to local football teams, as playing these might provide a trigger for disorder.

Previous research by the present author in pubs, also conducted in Glasgow, Scotland, found from both field observations and staff interviews that music was used strategically by operators to recruit customers, to retain them, and to mould their behaviour, in short to tempt them to spend more at the bar'. The negative consequences of this strategy were twofold; firstly increased bar sales meant an increased likelihood of alcohol-related disorder, secondly incidents of violent disorder were often centred on music, especially dancing (i.e. in the absence of dance-floors) and karaoke. However this previous research was limited in that pubs are also part of the Day-time (i.e. pre-midnight) Economy, and as such substance use patterns are likely to be less varied and arguably less extreme than in nightclubs (Glasgow's pub patrons were observed heading towards nightclubs at closing-time). Also, the role of music is less central to a pub's function. In contrast to pubs, in nightclubs sources of music such as karaoke (and jukeboxes) are less likely to be a feature of the sonic environment; however these (larger) venues are more likely to have designated dance-floors, live performers, purpose built sound-systems, light-shows etc. In particular the role of the $\mathrm{DJ}$ is likely to be more central to the nightclub environment ${ }^{20}$. This present research, conducted two years later in the same city centre, addresses these gaps in our knowledge. 
especialmente, juegos de luces, etc. En particular el papel de los DJs parece más central en el contexto de los clubes ${ }^{20}$. La presente investigación, llevada a cabo dos años más tarde en el mismo centro de la ciudad, trata de cubrir esas lagunas de conocimiento.

\section{MÉTODOS}

El método de investigación utilizado en este estudio fue la observación participante, que se llevó a cabo dentro de una muestra de ocho clubes nocturnos del centro de Glasgow entre febrero y marzo de 2006. Participaron dos grupos, de dos trabajadores cada uno, que comprendian a una estudiante y un estudiante como observadores en cada uno (cuyas edades oscilaban entre los 21-26 años), que visitaron los clubes seleccionados dos veces, una el viernes y otra el sábado, entre medianoche y las 3:00 a.m. (lo que hace un total aproximado de 100 horas de observación por lo que respecta al 'tiempo de beber'). Este procedimiento fue idéntico al utilizado en el estudio ${ }^{1}$ sobre los pubs musicales en 2004, siendo la única diferencia el tipo de locales observado.

Los ocho locales fueron elegidos entre los 70 clubes nocturnos de Glasgow, previa consulta a la policía local (Strathclyde Police), para que estuviera representada toda la gama de llamadas por desórdenes que reciben de dichos locales en el centro de la ciudad (es decir, que incluyera locales con niveles altos y bajos de delitos violentos registrados). Todos estos locales poseían el permiso denominado Entertainment License (es decir, podian servir alcohol hasta las 3:00 a.m. durante los fines de semana), cobraban admisión en la puerta y ofrecian música de consumo popular y promociones de baile regulares. Por tanto, la muestra excluía todos los clubes nocturnos sin baile (como los de comedia o striptease) locales pequeños como algunos clubes gay, ceilidhs (eventos tradicionales escoceses) o eventos 'rave' con entrada. Se asignó a cada local un pseudónimo apropiado.

Los observadores tomaron extensivas notas de campo sobre lo que habian visto en cada ocasión en que habian visitado los clubes. De vuelta a casa, después de cada sesión de observación, completaban también dos instrumentos de investigación (cuestionarios o listas de control). Estos instrumentos y esta metodología han sido ampliamente usados en Canadà por la profesora Kathryn Graham y sus colaboradores ${ }^{22,23}$ así como en la investigación previa realizada en Glasgow. El primero de estos instrumentos, 'Formulario 1', se utilizó para detallar el contexto de bebida dentro de cada club nocturno. Los ítems en el 'Formulario 1' hacian referencia a los tipos de música que se tocaban en cada local (fuente, género, artistas, etc.). El otro, 'Formulario 2', se utilizó para registrar cualquier acto de violencia presenciado. Los observadores no fueron informados de por qué habían sido seleccionados los ocho locales de la muestra, y ninguno de los equipos de investigación podía estar seguro de la política musical dentro de cada club hasta que la hubiera observado.

\section{METHODS}

The research method used for this study was participant observation which took place within a sample of eight Glasgow city centre nightclubs between February and May 2006. This involved two teams of two fieldworkers, comprising one female and one male student observer in each team (age range 21 - 26 years), visiting each selected nightclub twice, once on a Friday and once on a Saturday, midnight to 3.00AM (which approximates to 100 hours of observation taking into account 'drinking up time'). This procedure was identical to that used in the 2004 pub music study ${ }^{1}$, other than the type of venue being observed.

The eight venues chosen were selected from Glasgow's 70 nightclubs, in consultation with the local police (Strathclyde Police), to represent the range of call-outs to disorder which they received from such premises in the city centre (i.e. including some premises with high and some with low levels of recorded violent crime). These premises all held an Entertainment License (i.e. they served alcohol till 3.00 $\mathrm{AM}$ at weekends), charged admission at the door and offered mainstream 'high street', regular dance promotions. That is the sample excluded all types of non-dance nightclub (e.g. comedy clubs, strip clubs etc.) and niche dance venues such as Gay clubs, ceilidhs [traditional Scottish events] or ticketed 'rave' promotions. Each of the observed venues was assigned a suitable pseudonym.

Observers made extensive field-notes about what they had witnessed on each occasion that they visited a nightclub. On returning home after each observational session, they also completed two quantitative research instruments (questionnaires or checklists). These instruments and this methodology have been extensively used in Canada by Professor Kathryn Graham and colleagues ${ }^{22,23}$ as well as in the previous Glasgow pub music research'. The first of these, 'Form1', was used to detail the drinking environment within each nightclub. Items in 'Form1' enquired about the types of music being played at each venue (source, genre, artists, etc.). The other, 'Form2', was used to record any incidents of violence witnessed. Observers were not informed of why the eight nightclubs in the sample had been chosen, and none of the research team could be sure of the music policy within each venue until it had been observed. 


\section{RESULTADOS}

A pesar de haber sido seleccionados por su similar posicionamiento en el mercado, fue posible realizar una tipología de clubes nocturnos, afín a la de Purcell y Graham ${ }^{24}$ en Canadá, basada en la política musical registrada por los observadores en el 'Formulario 1'. Aunque muchos géneros musicales (como el reggae) o subgéneros (como el deep-house) fueron anotados por los observadores, para el propósito de este estudio los ocho clubes nocturnos de la muestra se clasificaron dentro de cuatro tipos de música distintos de acuerdo con aspectos únicos de su política musical de la siguiente manera:

- Locales 'Hardcore': Sólo en dos de los ocho locales se observó la utilización de mezclas de baile happy-hardcore, en 'Armageddon' y en 'Xanadu'. Como veremos, estos locales parecian atraer a la clientela más joven y camorrista.

- Locales 'Pop-pegadizo': Dos clubes nocturnos, 'Tropicana' y 'Sinatra's' tocaban, predominantemente, grandes éxitos (que en 2006 incluian a muchos artistas comerciales de rhythm and blues) o éxitos de ayer. Esta política parecia atraer a una clientela más general, que incluia a clientes mayores (de más de 30 años).

- Locales 'Urbanos': Dos clubes, 'Chocolate' y 'Idols' tocaban principalmente REB (incluyendo hip-hop y rap). El último de estos locales también contaba con una pequeña sala donde sonaba música house aunque, como lo explicó una de las observadoras, "parecía que la mayoría de clientes iban a 'Idols' por su sala grande de R\&B y solo aparecian en la pequeña para descansar" (Observadora femenina, Equipo B).

- Locales 'Mezclados': Un club, 'Rapture', se diferenciaba del resto de la muestra en que tenía dos pistas de baile, una con un estilo 'pop-pegadizo' (principalmente 'rock estudiantil'), mientras que la otra "se promocionaba ella misma como el club urbano preferido de Glasgow" (Observador masculino, Equipo B). La politica musical de 'mezcla' ('urbana'/'pop-pegadizo') de este 'super-club'24 se reflejaba en su clientela diversa, que variaba considerablemente entre sus dos pistas.

- Locales 'Old-skool rave' [variante del techno]: El último club, 'Saturn', difería bastante de los otros siete, en todas sus formas, ya que ponía música que resultó irreconocible a todos los observadores, sin canciones convencionales, y donde ninguna de las interpretadas allí (principalmente ambient o rave) tenía letras. Este local no atraía a los clubbers más jóvenes.

La Tabla 1 resume los puntos que definen esta tipología. También, la Tabla 1 muestra los artistas reconocidos de forma más frecuente por los observadores en el 'Formulario 1' y los estilos de baile dominantes observados, que junto al género musical, podían sumar un total de ocho observaciones (dos visitas a cada local por cuatro observadores).

\section{RESULTS}

Despite being chosen for their marketplace similarities, it was possible to produce a typology of nightclubs, similar to that of Purcell and Graham ${ }^{24}$ in Canada, based on music policy as recorded by observers on 'Form1'. Although many music genres (e.g. reggae) or sub-genres (e.g. deep house) were noted by observers, for the purposes of this paper, the eight nightclubs in the sample were classified into distinct four types based on unique aspects of their music policy as follows.

- 'Hardcore' venues: Only two of the eight nightclubs were ever observed playing happy-hardcore dance music mixes, 'Armageddon' and 'Xanadu'. As will be seen these venues seemed to attract a younger, rowdier clientele.

- 'Cheesy-pop' venues: Two nightclubs, 'Tropicana' and Sinatra's, predominantly played either chart music (which in 2006 included many commercial RetB artists) or oldies. This policy seemed to attract a more mainstream clientele, including many older patrons (aged 30+ years).

- 'Urban' venues: Two nightclubs, 'Chocolate' and 'Idols' played mainly RetB music (including hip-hop and rap). The latter of these venues also had a small side room playing house music, though as one observer put it "It seemed as if most patrons went into 'Idols' for the main R\&B room and just popped in here for a break" (Female observer, Team B).

- 'Mixed' venue: One nightclub, 'Rapture', differed from the others in the sample in that it had two floors, one of which had a 'cheesy-pop' style (mainly 'student rock'), while the other "advertised itself as "Glasgow's favourite Urban club" (Male, observer Team B). The 'mixed' ('urban' / 'cheesy-pop') music policy of this 'super-club'24 was reflected in a diverse clientele, varying greatly between these two floors.

- 'Old-skool rave' venue: The final nightclub, 'Saturn', was quite unlike the other seven, in all their guises, as this venue played no music by artists who was recognisable to any of the observers, there were no conventional songs and none of the music here (mainly ambient or rave) had any lyrics. This venue did not attract younger clubbers.

Table 1 summarises the defining points of this typology. Also shown on Table 1 are the artists most commonly noted by observers in 'Form1' and the dominant dancing styles observed, both of which, like music genre, could sum to a maximum of eight observations (from the two visits to each venue by the four observers). 
Tabla 1. Estilos de los clubes nocturnos

Table 1: Nightclub Styles

\begin{tabular}{|c|c|c|c|}
\hline $\begin{array}{l}\text { Club nocturno (tipo) / } \\
\text { Nightclub (type) }\end{array}$ & $\begin{array}{l}\text { Género Musical (n de observaciones) / } \\
\text { Music Genre (n of observations) }\end{array}$ & $\begin{array}{l}\text { Artista más escuchado (n de observaciones) / } \\
\text { Most Heard Artists (n of observations) }\end{array}$ & $\begin{array}{l}\text { Principales estilos de baile'( } \mathrm{n} \text { de observaciones) / } \\
\text { Main Dancing Styles (n of observations) }\end{array}$ \\
\hline $\begin{array}{l}\text { Armageddon } \\
\text { (hardcore) }\end{array}$ & Happy-hardcore (8) & $\begin{array}{l}\text { Mezclas del DJ (5) / DJ own mixes (5) } \\
\text { Pussycat Dolls (4) / Pussycat Dolls (4) } \\
\text { Will Smith (3) / Will Smith (3) }\end{array}$ & $\begin{array}{l}\text { Grinding (6) } \\
\text { Raving (6) } \\
\text { 'stomping' (3) }\end{array}$ \\
\hline $\begin{array}{l}\text { Xanadu } \\
\text { (hardcore) }\end{array}$ & $\begin{array}{l}\text { R\&tB (8) } \\
\text { Happy-hardcore (7) } \\
\text { Chart (6) }\end{array}$ & $\begin{array}{l}\text { Pussycat Dolls (7) } \\
\text { Kanye West (4) } \\
\text { Snoop Dogg (3) }\end{array}$ & $\begin{array}{l}\text { Raving (5) } \\
\text { 'sexy' (3) } \\
\text { Grinding (2) } \\
\text { School disco (2) }\end{array}$ \\
\hline $\begin{array}{l}\text { Sinatra's } \\
\text { (pop-pegadizo) / (cheesy-pop) }\end{array}$ & $\begin{array}{l}\text { Chart (8) } \\
\text { Oldies (8) } \\
\text { RetB (8) }\end{array}$ & $\begin{array}{l}\text { Kanye West (8) } \\
\text { Dolly Parton (5) } \\
\text { Queen (5) }\end{array}$ & $\begin{array}{l}\text { 'big groups' (2) } \\
\text { 'energetic' (2) } \\
\text { 'messing about' (2) }\end{array}$ \\
\hline $\begin{array}{l}\text { Tropicana } \\
\text { (pop-pegadizo) }\end{array}$ & $\begin{array}{l}\text { Chart (8) } \\
\text { Oldies (8) }\end{array}$ & $\begin{array}{l}\text { Dolly Parton (6) } \\
\text { Pussycat Dolls (5) }\end{array}$ & $\begin{array}{l}\text { 'wedding style' (4) } \\
\text { 'messing about' (2) }\end{array}$ \\
\hline $\begin{array}{l}\text { Rapture } \\
\text { (mezclado - urbano / pop-pegadizo) / } \\
\text { (mixed-urban / cheesy-pop) }\end{array}$ & $\begin{array}{l}\text { REtB (8) } \\
\text { Rock (7) } \\
\text { Chart (6) }\end{array}$ & $\begin{array}{l}\text { Pussycat Dolls (4) } \\
\text { Proclaimers (3) } \\
\text { Usher (3) }\end{array}$ & $\begin{array}{l}\text { Grinding (6) } \\
\text { Dirty-dancing (2) }\end{array}$ \\
\hline $\begin{array}{l}\text { Chocolate } \\
\text { (urbano) / (urban) }\end{array}$ & REB (7) & $\begin{array}{l}\text { Pussycat Dolls (5) } \\
\text { Kanye West (4) } \\
\text { Usher (4) }\end{array}$ & $\begin{array}{l}\text { Grinding (6) } \\
\text { 'sexy' (2) } \\
\text { Hip-hop nod (2) } \\
\text { 'modest' (2) }\end{array}$ \\
\hline $\begin{array}{l}\text { Idols } \\
\text { (urbano) / (urban) }\end{array}$ & $\begin{array}{l}\text { House (8) } \\
\text { R\&tB (8) }\end{array}$ & $\begin{array}{l}\text { Kanye West (6) } \\
\text { Nelly (4) } \\
\text { Snoop Dogg (4) }\end{array}$ & $\begin{array}{l}\text { Grinding (6) } \\
\text { 'sexy' (3) } \\
\text { Hip-hop nod (3) }\end{array}$ \\
\hline $\begin{array}{l}\text { Saturn } \\
\text { (old-skool rave) }\end{array}$ & $\begin{array}{l}\text { Ambient (5) } \\
\text { Rave (5) }\end{array}$ & $\begin{array}{l}\text { Ninguno 'conocido' (8) / } \\
\text { None 'Known' (8) }\end{array}$ & $\begin{array}{l}\text { 'modest' (5) } \\
\text { Raving (2) } \\
\text { 'energetic' (2) }\end{array}$ \\
\hline
\end{tabular}

'N.T.: Mantenemos los nombres originales por ser los utilizados por los clubbers de cualquier pais aunque traducimos aproximadamente el término para aquellos no familiarizados con la escena clubbing: Grinding (apretado/magreándose), raving (fuera de control), 'stomping' (picando de pies), 'sexy' (erótico), School disco (sujetando por detrás a la chica, con las manos en las caderas o culo), 'big groups' (en grupo),

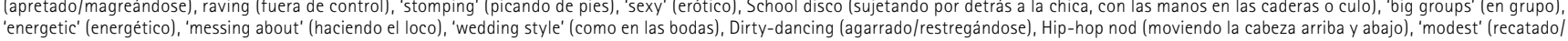
modesto).

Como puede verse en la Tabla 1 cada género musical parecía dictar los estilos de baile de la clientela. En primer lugar, los que podrian definirse como bailes de 'apareamiento', como el agarrado, y los estilos de baile 'sexys' y tipo dirty-dancing, con los que los clientes intentaban atraer a una pareja (es decir, 'morrearse'), parecía ser más comunes en los clubes 'urbanos'. En segundo lugar, la clientela de los clubes de 'pop-pegadizo' parecía dedicarse más a lo que puede denominarse como bailes 'divertidos', lo que, al contrario que en los locales urbanos, los observadores describían como 'interactuaciones' de diverso género (hombres y mujeres) entre 'grandes grupos' como, por ejemplo, 'compañeros de trabajo' o 'bodas' (despedidas de soltera). En tercer lugar, en los otros lugares, la clientela parecía bailar de acuerdo con la música, en vez de interactuando con los otros clientes como era el caso en los locales 'urbanos' y de 'poppegadizo', o formando parte de la 'escena' como estereotipos de un determinado género musical, tales como el 'raving' o baile descontrolado (observado tanto en los locales 'hardcore' como en los clubes 'old-skool rave', con movimientos de cabeza hip-hop y multitud de estilos minoritarios no mostrados en la Tabla 3 (como tocando la guitarra sin instrumento en 'Rapture', tipo country y western en 'Tropicana', salsa en 'Saturn' o break-dance en 'Idols' y 'Saturno').

La lista completa de los artistas anotados por los observadores era larga, pero limitada en términos de géneros musicales. Como puede verse en la Tabla 1, los mismos artistas (principalmente éxitos de R\&B) sonaban en clubes por lo demás muy distintos. Por ejemplo, la canción 'Gold-digger' de Kanye West se anotó, al menos una vez, en cada uno de los siete clubes donde los DJ ponían canciones (es decir, en el
As can been seen from Table 1 each music genre appeared to dictate patrons' dancing styles. Firstly what might be termed as 'mating' dances, such as grinding, 'sexy' and dirty-dancing dancing styles, where patrons may be attempting to attract partners (i.e. 'pulling'), appeared to be most common in the 'urban' nightclubs. Secondly the 'cheesy-pop' nightclubs' patrons appeared to engage in what might be termed as 'fun' dances, where, in contrast to the urban venues, observers described them as 'messing about' in (mixed gender) 'big groups' (e.g. 'office parties') or in a 'wedding style' (e.g. 'hen nights'). Thirdly, elsewhere patrons appeared to be dancing in appreciation of the music, rather than to interact with other patrons as was the case in the 'urban' and 'cheesy-pop' venues, or to be part of the 'scene' (i.e. 'scenesters'), such as 'raving' (observed in both the 'hardcore' venues and in the 'old-skool rave' nightclub), hip-hop nodding and numerous minority styles not shown in Table 3 (e.g. air-guitar in 'Rapture', Country \& Western in 'Tropicana', salsa in 'Saturn', or break-dancing in 'Idols' and 'Saturn').

The full list of artists noted by observers was lengthy, but narrow in terms of genre. As can be seen from Table 1, the same artists (mainly R\&B chart performers) appeared to be being played in otherwise very different nightclubs. For example the song 'Gold-digger' by Kanye West was observed, at least once, in all seven nightclubs where the DJs played any songs (i.e. the only nightclub where this popular song of 2006 was not heard was at the 'old-skool rave' venue 
Tabla 2. Clientela de los clubes nocturnos

Table 2: Nightclub Clientele

\begin{tabular}{|c|c|c|c|c|c|c|}
\hline \multirow{2}{*}{$\begin{array}{l}\text { Club Nocturno (tipo) / } \\
\text { Nightclub (type) }\end{array}$} & \multicolumn{6}{|c|}{$\begin{array}{c}\text { Estimaciones Observadas en cada club sobre la clientela (todos los porcentajes son la media de } 8 \text { observaciones) / } \\
\text { Observer Estimates of each nightclub's Clientele (all scores are the mean of } 8 \text { observations) }\end{array}$} \\
\hline & $\begin{array}{l}\mathrm{N} \text { de clientes } \\
\text { Nof patrons }\end{array}$ & $\begin{array}{l}\% \text { Mujeres } \\
\% \text { Female }\end{array}$ & $\begin{array}{l}\% \text { Menores de } 18 \text { años } \\
\% \text { Aged under-18 }\end{array}$ & $\begin{array}{l}\% \text { Mayores de } 30 \text { años } \\
\% \text { Aged over-30 }\end{array}$ & $\begin{array}{l}\% \text { Clase media } \\
\text { Middle class } \%\end{array}$ & $\begin{array}{l}\% \text { Minorias étnicas } \\
\% \text { Ethnic minority }\end{array}$ \\
\hline $\begin{array}{l}\text { Armageddon } \\
\text { (hardcore) }\end{array}$ & 246 & 50.6 & $46.9^{* * *}$ & 4.4 & $17.7^{* *}$ & $1.9^{*}$ \\
\hline $\begin{array}{l}\text { Xanadu } \\
\text { (hardcore) }\end{array}$ & 241 & 58.5 & $33.8^{* *}$ & 5.0 & 18.8 & 4.9 \\
\hline $\begin{array}{l}\text { Sinatra's } \\
\text { (pop-pegadizo) / (cheesy-pop) }\end{array}$ & 248 & 58.8 & $1.9^{*}$ & $35.6^{* * *}$ & $12.5^{*}$ & $1.1^{*}$ \\
\hline $\begin{array}{l}\text { Tropicana } \\
\text { (pop-pegadizo) / (cheesy-pop) }\end{array}$ & 252 & 60.6 & 4.6 & $34.8^{* * *}$ & 25.0 & $1.8^{*}$ \\
\hline $\begin{array}{l}\text { Rapture } \\
\text { (mezclado) / (mixed) }\end{array}$ & 251 & 52.5 & 8.6 & $4.4^{*}$ & 43.8 & $47.4^{* * *}$ \\
\hline $\begin{array}{l}\text { Chocolate } \\
\text { (urbano) / (urban) }\end{array}$ & 197 & 57.6 & 11.3 & 11.9 & 50.0 & $35.6^{* * *}$ \\
\hline $\begin{array}{l}\text { Idols } \\
\text { (urbano) / (urban) }\end{array}$ & 258 & 60.6 & 12.5 & 7.5 & 50.0 & 22.1 \\
\hline $\begin{array}{l}\text { Saturn } \\
\text { (old-skool rave) }\end{array}$ & $154^{* * *}$ & $45.0^{* *}$ & $0^{* *}$ & 16.3 & $68.7^{* *}$ & 6.8 \\
\hline Mean & 231 & 55.5 & 14.9 & 15.1 & 34.4 & 15.2 \\
\hline
\end{tabular}

${ }^{*} p>0.05,{ }^{* *} p>0.01,{ }^{* * *} p>0.001$ (mediante la prueba t comparando la media para cada club con la media combinada de los otros siete clubes)

${ }^{*} p>0.05,{ }^{* *} p>0.01,{ }^{* * *} p>0.001$ (by t-test comparing the mean for each nightclub with the mean for the other seven combined).

único sitio donde no se escuchó esta popular canción del 2006 fue en el local 'old-skool rave', 'Saturn', donde no se tocaban canciones). Como argumentaremos después, esta política podía estar encaminada a conseguir una clientela más amplia, como parte de un circuito, quizás. Las diferencias observadas en la clientela, que cada uno de estos ocho clubes atraía, se indica en la Tabla 2, que suministra los porcentajes medios de cada una de las variables, atendiendo a las características de la clientela del 'Formulario 1, en la que cada observador proporcionó una puntuación por visita a cada local (un total de ocho observaciones por cada variable).

En la Tabla 2 puede verse que los dos locales 'hardcore' atraían a menores de 18 años (edad legal para la compra de alcohol en Escocia), especialmente 'Armageddon', donde sólo sonaba este tipo de música de baile. Sin embargo, este local 'hardcore' resultaba poco atractivo a los estudiantes-observadores, como ilustran las siguientes notas de campo:

"El tipo de cosas happy-hardcore que tocaban parecía atraer al tipo equivocado de clientes lo cual es una pena, porqué si no hubiera sido por los 'hooligans' y la música de mierda podria resultar un club bastante decente". (Observador masculino, Equipo B, 'Xanadu').

"Este es el tipo de club donde la gente, supuestamente, va 'por la música' así que prestan poca atención a la decoración para crear un cierto ambiente. El problema, sin embargo, es que la música es una mierda y que la versión happy-hardcore de los Bros 'I owe you nothing' no arregla el hecho de que el local es un antro!" (Observadora femenina, Equipo B, 'Armageddon').

Aunque en cierta manera subjetiva, la queja final de la observadora femenina resulta informativa. Una escala en el 'Formulario 1' medía la 'suciedad' (entre 0 y 29), y esté club puntuó el máximo (la puntuación media de las ocho observaciones fue 24.2 , mientras que la media para los otros siete clubes combinados fue de $15.7, t=3.475: p=0.007$ ).
'Saturn' which played no songs at all). As will be discussed later, this policy may have been to attract a wide clientele, perhaps as a part of a circuit. The observed differences in clientele, that each of these eight nightclubs attracted, is indicated by Table 2, which provides the mean scores for all variables measuring patron characteristics on 'Form1', with each observer providing one score per visit to each premises (a total of 8 observations per variable).

From Table 2 it can be seen that the two 'hardcore' venues attracted many under-18s (the legal age for alcohol purchase in Scotland), especially 'Armageddon' which only played this style of dance music. However, this 'hardcore' venue was less than attractive to the student observers, as the following field-notes illustrate.

\footnotetext{
"The type of happy-hardcore stuff they play seems to attract the wrong type of customers which is a shame because if it wasn't for all the neds [Scottish 'hooligans'] and the crap music it could actually be a decent enough club." (Male Observer Team B, 'Xanadu')

"This is the kind of club where people allegedly 'go for the music' so little attention was paid to the décor of the club or trying to create a certain atmosphere. The problem however, is that the music is crap and a happy-hardcore version of Bros 'I owe you nothing' doesn't make up for the fact that the place is a hole!" (Female Observer Team B, 'Armageddon')
}

Though somewhat subjective, the final complaint by the female observer above is informative. A scale on 'Form1' measured 'dirtiness' (range 0 to 29), and this nightclub scored the highest (mean score across eight observations 24.2, against a mean score 15.7 for the other seven nightclubs means combined, $t=3.475: p=0.007)$. However, unlike the 
Sin embargo, al contrario que los observadores, pero en línea con las predicciones de Homel y Tomsen ${ }^{19}$ (ver Introducción), a los seguidores del happy-hardcore la atracción de este género musical parecía invalidar dicha característica negativa del 'Armageddon'.

Al contrario que en los locales 'hardcore', como muestra la Tabla 2, los dos locales de 'pop-pegadizo' parecían satisfacer una gama más amplia de edades tocando, por consiguiente, más canciones de ayer que otro tipo de clubes. Se ofrecian en especial temas de películas, que incluian el 'Nine to five' de Dolly, Parton, el 'Don't stop me now' de Queens de la banda sonora de Shaun of the Dead (Tabla 1), temas de Footloose o Grease, y el 'Stuck in the middle with you', de una escena particularmente violenta de Reservoir Dogs.

"Amplia variedad de edades en el local. Por lo menos una despedida de soltera en acción. El vestuario de los hombres va desde trajes a camisetas y deportivas (bambas). Para las mujeres, lo más común es faldas cortas o vestidos, etc., aunque como para algunas era una despedida de soltera, había grupos que llevaban también cuernos de diablesa o orejas y cola de conejitos. Parecía que estuvieran pasando un casting para la película El diario de Bridget Jones". (Observador masculino, Equipo A, 'Tropicana').

Los locales 'urbanos' parecian atraer una clientela más diversa desde el punto de vista étnico que los otros clubes. Esto era especialmente obvio en 'Rapture', el club 'mezclado' ('urbano'/'pop-pegadizo') con dos pistas, en una de las cuales sonaba RetB y en la otra 'rock estudiantil'.

"La pista de arriba resultaba acogedora a los estudiantes: bastante oscura y cargada de humo con asientos alrededor de su parte más exterior. No estaba decorada con ningún estilo en particular, probablemente porque los clientes estaban demasiado borrachos para darse cuenta. Parecía bastante desastrada comparada con la pista de abajo. La de abajo era la pista 'Urbana' y en su mayoría los clientes eran negros o del sudeste asiático (hombres en su mayoria). Había una atmósfera con una cierta actitud negativa aunque no hostil. La mayoria sólo jugaba un papel. El código de vestimenta en esta pista era 'home boys'. Mucho 'brillo' [bisutería ostentosa]". (Observadora femenina, Equipo B, 'Rapture').

Más que ningún otro, el local 'old-skool rave', 'Saturn', parecía venderse en su política musical, quizás no resulte sorprendente, dado lo distinta que resultaba su música comparada con la de los otros siete locales. Cualquiera que fuera la razón, la clientela de este club resultó ser la más 'clase media' y 'masculina' de la muestra (Tabla 2).

"Exceptuando una promoción de bebidas, no se promocionaron otro tipo de ofertas o tratos especiales. Había folletos en la mayoria de las mesas y pósters en las paredes sobre eventos a punto de celebrarse (música electrónica de baile) anunciando fechas y DJs invitados. 'Saturn' parecía promocionar más la música que depender de las promociones de bebidas" (Observador masculino, Equipo B, 'Saturn').

"...a diferencia de los otros clubes, la clientela era de mayor edad, bebía menos y estaba alli por la música en vez de para emborracharse o mojar (conseguir una pareja sexual), así que la atmósfera era más relajada. Creo que la música ambiental funky también ayudaba a esta atmósfera. Todos los clientes contentos, mucho contacto visual y sonrisas. Atmósfera cari- observers, but in line with prediction of Homel and Tomsen ${ }^{19}$ (see Introduction), to a happy-hardcore fan-base the attraction of this music genre appeared to have over-ridden this negative feature at 'Armageddon'.

In contrast to the 'hardcore' venues, as shown by Table 2 , the two 'cheesy-pop' venues seemed to cater for a greater age range, accordingly playing more oldies than other nightclub types. Movie themes were a particular feature, including Dolly Parton's 'Nine to five', Queen's 'Don't stop me now', from the soundtrack of Sean of the Dead (see Table 1), the themes to Footloose or Grease, and Stealer's Wheel's 'Stuck in the middle with you,' from a particularly violent scene in Reservoir Dogs.

"Very large age range in the place. At least one hen party [bachelorette party] happening. Dress for men ranged from suits to t-shirts and trainers [sneakers]. For women, your usual short skirts / dresses etc., although as it was a hen night for some, group were wearing devil horns or bunny ears and tail. It looked like auditions were being held for Brigit Jones's Diary film." (Male Observer Team A, 'Tropicana')

The 'urban' venues seemed to attract a more ethnically diverse clientele than the other nightclubs. This was most obvious in 'Rapture', the 'mixed' ('urban' / 'cheesy pop') nightclub with two floors, one of which played R\&B, the other 'student rock'.

"The top floor was student friendly: quite dark and very smoky with seating around the outside. It wasn't really decorated in any particular style, probably as most patrons were too drunk to notice. It looked fairly shabby compared to the bottom floor. This floor was the 'Urban' floor and was represented by mostly Black or [South] Asian patrons (mostly male). There was an atmosphere of attitude but not in a hostile way. Most people just wanted to look the part. Dress code for this floor was definitely 'home boys'. Lots of 'bling' [ostentatious jewellery]." (Female Observer Team B, 'Rapture')

More than any other, the 'old-skool rave' venue 'Saturn' appeared to sell itself on its music policy, perhaps unsurprisingly so, given how different this music was to that of any of the other seven venues. Whatever the reason, this nightclub's clientele appeared to be both the most 'middle class' and male in the sample (see Table 2).

"Apart from the one drink promotion, no special offers or deals were advertised. There were flyers on most of the tables and posters on the walls on the way in for upcoming [electronic dance music] nights and guest DJs. 'Saturn' seems to promote its music rather than rely on drink promotions" (Male Observer Team B, 'Saturn')

"...unlike the other clubs, patrons were older, drinking less and there for the music rather than to get fucked or pull [attract a sexual partner], so atmosphere much more relaxed. I think the more ambient funky music helped the atmosphere too. All patrons very friendly lots of eye contact and smiling. Lovedup atmosphere, everyone really enjoying the music and danc- 
ñosa cargada de buen rollo, todo el mundo disfrutando de la música y bailando con la gente a su alrededor, desconocidos 0 no". (Observadora femenina, Equipo A, 'Saturn').

Como ilustra la segunda nota de campo, la política musical de 'Saturno' no sólo ejercía una influencia en su clientela sino que también influenciaba su comportamiento una vez dentro del local. Los clientes de 'Saturn' consumian menos alcohol, pero más drogas ilegales (éxtasis), y consecuentemente parecian más amigables y mucho menos turbulentos como era el caso en cualquier otro de los clubes.

"Atmósfera simpática y amigable hasta cierto punto, porque sabes que todos los clientes están empastillados (de éxtasis) y no te darían ni los buenos dias si estuvieran limpios. Aunque un tipo me dio sus dos latas de Heineken (cerveza) gratis, porque llevaba una pulsera que molaba. La única forma en que ALGUIEN, en cualquiera de los otros clubes, te hubiera dado sus copas hubiera sido lanzándotelos encima con ira". (Observador masculino, Equipo A, 'Saturno').

La Tabla 3 detalla los comportamientos de los clientes, tal como se registraron en el 'Formulario 1' y el 'Formulario 2' (incidentes agresivos presenciados). Como puede verse en la Tabla 3, los dos clubes 'hardcore' presentaron los niveles más altos de borracheras presenciadas. Por el contrario el club 'old-skool rave' fue el único local donde se estimó a sólo una minoría de clientes como borrachos. Los dos clubes 'hardcore' también mostraron niveles altos de consumo de drogas ilegales, aunque no fueron tan altos como los del club 'old-skool rave'. El nivel de consumo de drogas ilegales en los clubes de 'pop-pegadizo' y 'urbanos' fue bajo, mientras que el consumo de alcohol variaba entre ellos siendo en conjunto menor que en los locales 'hardcore' pero mucho más alto que en el club 'old-skool rave'.

Como podría esperarse el número de incidentes violentos presenciados por los observadores se correspondía de cerca tanto con el género musical como con el consumo de alcohol. Como puede verse en la Tabla 3, se presenciaron más ing with the people around them, strangers or not." (Female Observer Team A, 'Saturn')

As the second field-note above illustrates, not only did the music policy in 'Saturn' influence clientele, it also influenced their behaviour once patrons were inside. 'Saturn' patrons consumed less alcohol, but more illegal drugs (ecstasy), and consequently they appeared friendlier and much less disorderly than was the case in any of the other nightclubs.

"Nice friendly atmosphere to a point, because you know that most of the patrons are all pilled-up [on ecstasy] and wouldn't give you the time of day when clean. Although one guy gave me his two free cans of Heineken [lager], because he had a wristband, which was cool. The only way anyone from ANY of the other clubs would give you their booze would be if threw it at you in anger." (Male Observer Team A, 'Saturn' - emphasis his)

Table 3 details patrons' behaviours, as recorded on 'Form1' and 'Form2' (aggressive incidents witnessed). As can be seen from Table 3, the two 'hardcore' nightclubs had the highest levels of observed drunkenness. In contrast the 'old-skool rave' nightclub was the only venue where only a minority of patrons were estimated to be drunk. The two 'hardcore' nightclubs also had elevated levels of illegal drug use, though these were not as high as in the 'old skool-rave' nightclub. Levels of illegal drug use were low in the 'cheesypop' and 'urban' nightclubs, while alcohol use varied between these, overall being lower than in the 'hardcore' venues, but much higher than in the 'old-skool rave' nightclub.

As might be expected the number of incidents of violent disorder witnessed by observers was closely related to both musical genre and alcohol consumption. As can be seen in Table 3, more violent incidents were witnessed in the two

Tabla 3. Comportamientos de los clientes

Table 3: Patrons Behaviours

\begin{tabular}{|c|c|c|c|c|c|c|}
\hline \multirow{2}{*}{ Club nocturno / Nightclub } & \multirow{2}{*}{$\begin{array}{l}\% \text { de clientes borrachos } \\
\text { observado / } \\
\text { Observed\% of patrons drunk }\end{array}$} & \multirow{2}{*}{$\begin{array}{c}\% \text { de clientes que parecian no } \\
\text { haber consumido drogas / } \\
\text { Observed \% patrons obviously } \\
\text { on drugs }\end{array}$} & \multirow{2}{*}{$\begin{array}{l}\text { Tensión sexual observada. } \\
\text { Escala }(0-27) / \\
\text { Observed Sexual Tension } \\
\text { scale }(0-27)\end{array}$} & \multirow{2}{*}{$\begin{array}{l}\text { Incidentes de agresión } \\
\text { observados / } \\
\text { Observed Incidents of } \\
\text { Aggression }\end{array}$} & \multicolumn{2}{|c|}{ Registro policial / Police Records } \\
\hline & & & & & $\begin{array}{l}\text { Delitos violentos / } \\
\text { Violent Crimes }\end{array}$ & $\begin{array}{l}\text { Llamadas por desórdenes / } \\
\text { Disorder call-outs }\end{array}$ \\
\hline $\begin{array}{l}\text { Armageddon } \\
\text { (hardcore) }\end{array}$ & $78.5^{* * *}$ & $41.3^{*}$ & 16.0 & 11 & 102 & 93 \\
\hline $\begin{array}{l}\text { Xanadu } \\
\text { (hardcore) }\end{array}$ & $78.1^{*}$ & 28.1 & 15.8 & 8 & 76 & 76 \\
\hline $\begin{array}{l}\text { Sinatra's } \\
\text { (pop-pegadizo) / (cheesy-pop) }\end{array}$ & 69.4 & 5.6 & 12.9 & 4 & 35 & 77 \\
\hline $\begin{array}{l}\text { Tropicana } \\
\text { (pop-pegadizo) / (cheesy-pop) }\end{array}$ & 57.5 & $0.3^{*}$ & 12.5 & 4 & 72 & 35 \\
\hline $\begin{array}{l}\text { Rapture } \\
\text { (mezclado) / (mixed) }\end{array}$ & 58.1 & 4.4 & $17.0^{* *}$ & 0 & 48 & 46 \\
\hline $\begin{array}{l}\text { Chocolate } \\
\text { (urbano) / (urban) }\end{array}$ & 63.1 & 2.9 & 13.1 & 2 & 42 & 39 \\
\hline $\begin{array}{l}\text { Idols } \\
\text { (urbano) / (urban) }\end{array}$ & 68.8 & $8.1^{*}$ & 16.4 & 5 & 25 & 51 \\
\hline $\begin{array}{l}\text { Saturn } \\
\text { (old-skool rave) }\end{array}$ & $38.8^{* * *}$ & $50.0^{* * *}$ & $6.1^{* * *}$ & 0 & 0 & 6 \\
\hline Mean & 64.0 & 17.8 & 13.7 & 4.25 & 50 & 52.9 \\
\hline
\end{tabular}

${ }^{*} p>0.05,{ }^{* *} p>0.01,{ }^{* * *} p>0.001$ (por t-test comparando la media de cada club con la media combinada de los otros siete clubes).

${ }^{*} p>0.05,{ }^{* *} p>0.01,{ }^{* * *} p>0.001$ (by t-test comparing the mean for each nightclub with the mean for the other seven combined). 
incidentes violentos en los dos locales 'hardcore', mientras que no se vio ninguno en el club 'old-skool rave'. Este era el mismo patrón de incidentes violentos registrado por la policía a lo largo del año anterior (2005). La columna final de la Tabla 3 suma el número total de delitos violentos recogidos en cada local y el número total de llamadas a la policía por incidentes y desórdenes en cada uno. De nuevo, los dos locales 'hardcore' obtienen la puntuación más alta y el club 'old-skool rave' la más baja, ya que no se registró ningún incidente violento.

Aunque, al igual que en 'Saturn', no se presenciaron incidentes durante las cuatro visitas de los equipos a 'Rapture' (ver Tabla 3), a diferencia de en 'Saturn' los observadores apreciaron que era más fácil que ocurriera, como resultado de la 'mezcla' de comportamientos de la clientela del local. En realidad, este club de dos pisos fue considerado uno de los más turbulentos de la muestra (es decir, de acuerdo con las cifras de la policía -que los observadores desconocian durante el trabajo de campo), aunque por causas distintas en cada uno de los pisos. Especificamente, el nivel de actividad sexual en el piso 'urbano' era el más alto de la muestra, medido en una escala (de 0 a 27) en el 'Formulario $1^{\prime 22,23}$ los niveles registrados de tensión sexual (creados sumando las tres escalas que median 'acoso a las mujeres', 'actividad sexual' y 'morreos' respectivamente. Alpha de Cronbach $=0.709$ ).

Estos comportamientos sexuales de riesgo parecian estar relacionados con los estilos de baile de 'apareamiento' que predominaban en esta pista 'urbana' (ver Tabla 1 y las notas de campo previas en relación a este club). Como se muestra en la Tabla 3, en contraposición a 'Rapture', los niveles más bajos de 'tensión sexual' se observaron en 'Saturno', el local 'old-skool rave'. En la pista de 'pop-pegadizo' de 'Rapture', la política musical parecía fomentar la borrachera ruidosa, como ilustran las siguientes notas de campo.

"Incluso el nivel de borrachera era variado. En la pista de arriba [pop-pegadizo] la gente estaba mucho más borracha que en la de abajo, pero quizás en la de abajo [urban] era más importante parecer vacilón que buscar bronca o bailar de forma estúpida como lo hacian arriba". (Observadora femenina, Equipo B, 'Rapture').

"Arriba era como otro club, más tranquilo pero más bullicioso, sonaba música rock. La gente tenía una pinta más de estudiante rockero. El DJ tocaba Duelling Banjos [de la banda sonora de la película Deliverance] y la gente se volvía loca dando patadas en el suelo y gritando 'Easy! Easy!' y empezando a saltar unos sobre las espaldas de los otros mientras que el auxiliar [seguridad] simplemente miraba". (Observadora Femenina, Equipo A, 'Rapture').

Este incidente también ilustra como un simple cambio de disco puede suponer un impacto en los niveles de desorden de la multitud. Estos cambios en tempo podían ser largos, ya que la política musical de cada club no era estática, sino que se desplegaba conforme pasaba la noche (aunque se utilizaran listas), como ilustra la siguiente observación hecha en 'Xanadu':

"La primera impresión no fue mala, durante los primeros $30 \mathrm{o}$ 45 minutos había luz y la música era corriente pero entonces a las 00:15am se apagaron las luces (o se oscurecieron mucho) 'hardcore' venues, while none were witnessed in the 'oldskool rave' nightclub. This was the same pattern as for violent incidents known to the police during the whole of the previous calendar year (2005). The final column in Table 3 sums the total number of violent crimes recorded at each premises and the total number of police call outs to incidents of disorder at each. Again the two 'hardcore' venues score highest and the 'old-skool rave' nightclub the lowest, where zero violent crimes were recorded.

Although, like 'Saturn', no violent incidents were witnessed during the research teams' four visits to 'Rapture' (see Table 3), unlike 'Saturn' this was felt by observers to been more down to chance, than as a result of this 'mixed' venue's patrons' behaviours. Indeed this two floored nightclub was felt to be one of this most disorderly in the sample (i.e. in line with the police figures - which observers were unaware of during fieldwork), albeit for very different reasons on each floor. Specifically, the level of sexual activity on the 'urban' floor was the highest in the sample, as measured by a scale (from 0 to 27) on 'Form1'22, 23 recording levels of 'sexual tension' (created by summing three scales measuring 'harassment of females', 'sexual activity' and 'pulling' respectively, Cronbach's alpha $=0.709$ ). These risky sexual behaviours appeared to be related to the 'mating' dancing styles which predominated on this 'urban' floor (see Table 1 and previous field-notes relating to this nightclub). As is shown on Table 3, in contrast to 'Rapture' by far the lowest levels of 'sexual tension' were observed in the 'old skool rave' venue 'Saturn'. Meanwhile, on the cheesy-pop floor of 'Rapture' the music policy seemed to encourage drunken rowdiness, as is illustrated in the following fieldnotes.

"Even the level of drunkenness was varied. On the top floor [cheesy-pop] people were far more drunk than the bottom floor, but maybe the bottom floor [urban] it was more important to look cool and not mess about or dance in a stupid way as they were [up]stairs." (Female Observer Team B, 'Rapture')

"Upstairs like a different club, quieter but more boisterous, playing rock music. More student rocker style crowd. DJ played Duelling Banjos [from the Deliverance movie soundtrack] and crowd went mad stamping their feet and shouting 'Easy! Easy!' and then started jumping on each others' backs while steward [security] looked on." (Female Observer Team A, 'Rapture')

The above incident also illustrates how simply changing a record can impact upon levels of crowd disorder. Such changes in tempo could be long lasting, as the music policy of each nightclub was not static, but developed as the night wore on (i.e. set lists were being used), as is illustrated in the following observation at 'Xanadu'.

"First impressions were not too bad as for the first $30-45$ minutes it was light and the music was mainstream but then at 00:15 AM the lights went off (or dimmed a lot!) and the happy 
y empezó el happy harcore. La atmósfera cambió instantáneamente y devino más intimidante." (Observadora femenina, Equipo B, 'Xanadu').

Esta nota de campo fue hecha por una observadora a la que disgustaba especialmente el happy-hardcore (ver nota de campo anterior) e ilustra como los observadores no fueron inmunes a los efectos de la política musical de cada local. Aunque los ocho clubs nocturnos escogidos para este estudio excluian a propósito cualquier local que los observadores frecuentaran (para asegurar que sus observaciones fueran discretas), sus diferentes niveles de familiaridad (o mejor, su falta de familiaridad) con algunos tipos de locales de música en los que se encontraron impactó de forma inevitable la naturaleza de sus observaciones (por ejemplo, en términos de con qué comparaban personalmente cada local, durante la primera ronda de observaciones -aunque durante la segunda visita a cada uno de ellos, tendieron a restringir las comparaciones a los otros clubes de la muestra).

"En términos de música y clientela esta sería la típica salida del sábado noche, baile / música pegadiza. Hasta el momento (comparado con 'Idols' [urbano] y 'Armageddon' [hardcore]) había mayor variedad de grupos de edad. Y, hasta cierto punto, una atmósfera más relajada, como la de los clubes de mi pueblo [población industrial en el norte de Inglaterra]." (Observador masculino, Equipo A, 'Sinatra's').

"Creo que yo tenía una ventaja respecto a Andy [observador masculino que hizo la nota de campo de más arriba], ya que yo era un adolescente a principios de la década de 1990, y por el sitio de donde venía [población costera en Escocia, escenario de una grave muerte en un club por drogas en 1994] así que estaba acostumbrado a la música rave y a los hooligans empastillados. Andy parecía un conejo atrapado bajo los focos. La gente era peligrosa y te podian patear la cabeza si los mirabas de forma inadecuada. Como si hubiéramos vuelto a la disco del colegio [con muchos menores de 18 años]. Con mucho morreo al azar, gente bailando rave y desmayándose, haciendo carotas, cantos de "estamos aquí, estamos aquí, jódete, vete". Muchos empujones, juegos violentos, bebidas derramadas. Una atmósfera muy tensa. Muchos auxiliares [personal de seguridad], pidiéndole constantemente a la gente que se largara. La pista de baile peligrosa con todas las riñas, las peleas, y los saltos y empujones al ritmo de la música... Es imposible saber qué es un incidente agresivo y qué baile." (Observadora femenina, Equipo A, 'Armageddon').

Como ilustra la segunda nota de campo, al no iniciado, este tipo de local 'hardcore' podía resultarle muy intimidante. Junto con el tipo de clientela que este género musical parece atraer, la naturaleza frenética de la música y del baile asociado (por ejemplo, saltos y empujones) representaban un factor de riesgo observado para los desórdenes. Esto se correspondería con el estudio previo de pubs ${ }^{1}$, donde además de los estilos de baile dándose golpes en plan sexy o agresivo, comunes a ambos estudios, el club nocturno parecía tener un riesgo inherente extra debido al gran tamaño de este tipo de locales, que hace que los incidentes y desórdenes sean más difíciles de identificar (ver u oír), como ilustra la nota de campo previa. Con esta idea en la cabeza, resultó interesante anotar que los DJs (podría decirse que la fuente de mucho de este desorden) hicieran doblete en estos clubes nocturnos como 'ojos y orejas' del personal de seguridad. Como muestra hardcore began. The atmosphere changed instantly and became more intimidating." (Female Observer Team B, 'Xanadu')

The above field-note was made by the observer who had a particular dislike of happy-hardcore (see earlier fieldnote) and illustrates how observers were not immune to the effects of the music policy of each venue. Although the eight nightclubs chosen for this study purposively excluded any premises which observers themselves frequented (to ensure that their observations were unobtrusive), their differing levels of familiarity (or more often their lack of it) with the types of music venues in which they found themselves inevitably impacted upon the nature of their observations (e.g. in terms of personal yardsticks to measure each venue against, during the first round of observations - though by their second visit to each they tended to restrict comparisons to the other nightclubs in the sample).

"In terms of music and patrons this is your average Saturday night out, dancing / cheesy music. So far (compared to 'Idols' [urban] and 'Armageddon' [hardcore]) there was a larger age range. More relaxed atmosphere to a point, much like the clubs back home [North England industrial town]." (Male Observer Team A, 'Sinatra's')

"I feel I had an advantage over Andy [the male observer who made the above field-note] as I was a teenager in the early 1990s and come from [a Scottish seaside town, scene of a high profile club-drug death in 1994] so I am used to rave music and pilled-up neds [Scottish 'hooligans']. Andy was like a rabbit caught in the headlights. People were rough and would have kicked your head in if you looked at them the wrong way. Like being back at school disco [many under-18s]. Lots of random snogging, rave dancing people passed out, gurning, chants of "here we here we here we fuckin' go". Lots of pushing, horseplay, spilled drinks. Very tense atmosphere. Lots of stewards [security staff], people constantly being asked to leave. Dance floor dangerous with brawls, fights and moshing... It's impossible to know what is an aggressive incident and what is dancing." (Female Observer, Team A 'Armageddon')

As the second field-note above goes on to illustrate, to the uninitiated this type of 'hardcore' venue could appear intimidating. As well as the type of clientele this music genre seemed to attract, the frenetic nature of the music and associated dancing (e.g. moshing) represented an observed risk factor for disorder. This would appear to be in line with the previous pub study'. As well as the 'sexy' or aggressive (bumping) dancing styles, common to both studies, the nightclub environment also seemed to have some inherent extra risks owing to the larger scale of these venues, making disorderly incidents harder to spot (hear or see), as the previous fieldnote also illustrates. With this in mind, it was interesting to note that DJs (arguably the source of much of this disorder) were doubling in these nightclubs as the 'eyes and ears' of the security staff. As the field-notes below illustrate, from their booths DJs were in a unique position to spot fights on the dance-floor. When DJs decided a security intervention 
la nota de campo más abajo, desde sus cabinas los DJs se encuentran en una posición única para identificar peleas en la pista de baile. Cuando un DJ decidia que se necesitaba una intervención de seguridad bajaba el volumen de la música y suministraba información verbal al personal de seguridad del local sobre la ubicación de la pelea. Estas intervenciones de los DJs también alertaron a los observadores de la presencia de desórdenes violentos dentro de los locales.

"El DJ dijo por el micro algo del estilo "pelea frente a la cabina del DJ". Tres auxiliares [seguridad] corrieron hacia la pista. Nosotros salimos a la carrera tras ellos [para observar la pelea]." (Observadora femenina, Equipo A, 'Sinatra's').

"Después de dos avisos del DJ de que había una pelea, S1 [auxiliar masculino] corrió detrás de P2 [cliente agresivo] y sus amigos..." (Observador masculino, Equipo A, 'Xanadu').

"Una vez que ellos [los que se peleaban] hubieron salido, el DJ dijo algo sobre "si queréis pelea, salid fuera" ya que "no hay lugar para ello dentro del club" y también que "vaya noche de locos que era". (Observadora femenina, Equipo A, 'Xanadu').

Como implica el comentario final del DJ en la nota de campo anterior, los animadores tienen evidentemente que equilibrar su papel de asistir en la seguridad mientras mantienen a los clientes contentos. Sin embargo, mantener a los clientes contentos podría ser en si misma una tarea para expertos. Por un lado, las multitudes entretenidas es menos probable que causen problemas. Por otro, esto puede llevar a los clientes a consumir más alcohol, lo que podria incrementar la posibilidad de desórdenes. Adicionalmente, algunos DJs parecian estar más dedicados a la venta directa de bebidas, publicitando verbalmente algunos productos o el mismo alcohol a través del micro, y utilizando a menudo un lenguaje que no pasaría ningún código de publicidad responsable de la industria alcoholera (por ejemplo, el del organismo de control del Portman Group ${ }^{25}$ de la industria del Reino Unido).

"DJs gritando sin parar "¿donde estáis todos, alcohólicos?", "¿quién ha venido a emborracharse?" y "venga, todos a la pista", etc., etc... DJs haciendo referencias continuamente a emborracharse y divertirse. DJs derramando champaña de una botella, desde el escenario, sobre las bocas de las mujeres". (Observadora femenina, Equipo A, 'Tropicana').

"El DJ mencionándola continuamente [la marca de un alcopop] "bebida energética como el Lucozade", "no sabe a pis", "es el futuro" y "es una bebida energética que mezclada con vodka os caeréis de culo"... El DJ haciendo continuas referencias al alcohol "¿quién ha salido a pillar la mona?", "¿dónde están todos los borrachuzos de Glasgow?", "¿quién se ha pasado el día bebiendo?" y "a ver, los que estáis puestos hasta el culo, gritad". (Observadora femenina, Equipo A, 'Xanadu').

La función final de la música en la experiencia del club nocturno es marcar el final de la noche (el cese del baile y de la venta de bebidas) y controlar la subsiguiente salida de los clientes. Al igual que hemos visto al comenzar la noche con el incremento en el tempo (o el cambio de género, por ejemplo a hardcore, como señalaba una nota previa) pero a la inversa, en los minutos antes del cierre se observó que algunos clubes reducian progresivamente el tempo de la música (que indica de nuevo el uso de listas determinadas, ver Hadfield ${ }^{26}$ ). El cambio producido en la atmósfera también indica a los regulares was necessary they would lower the volume of the music and provide verbal information to the venue's security staff on the location of the fight. Such interventions by DJs also alerted the observers to the presence of violent disorder within a venue.

"DJ said something over the microphone to the effect "fight in front of the DJ box". Three stewards [security] ran onto the dance floor. We legged it after them [to observe the fight]." (Female Observer Team A, 'Sinatra's')

"After two calls from the DJ that a fight was occurring S1 [male steward] ran behind $\mathrm{P} 2$ [aggressive patron] and his friends..." (Male Observer Team A, 'Xanadu')

"Once they [the fighters] had left the DJ commented something about "if you want to fight" to "go outside" as "there was no place for it in the club" and what a "mad night it was". (Female Observer Team A, 'Xanadu')

As the final comment by the DJ in the previous fieldnote implies, entertainers clearly had to balance their roles of assisting security whilst keeping patrons happy. However keeping the patrons happy could in itself be a skilled task. On the one hand entertained crowds may be less likely to cause trouble. On the other hand this may lead patrons to consume more alcohol, perhaps increasing the likelihood of disorder. Additionally, some DJs appeared to be involved in more direct drinks marketing by verbally advertising certain products or alcohol per se over the microphone, often using language which would not pass any drinks industry responsibility advertising codes (e.g. that of the UK drinks industry's watchdog the Portman Group ${ }^{25}$ ).

"DJs constantly shouting "where are all you alcoholics", "who's here to get drunk?" and "everyone on the dancefloor" etc. etc... DJ constantly making references to getting drunk and having fun. DJ pouring champagne from bottle into women's mouths from the stage." (Female Observer Team A, 'Tropicana')

"DJ constantly mentioned it [an alcopop brand name] "energy drink like Lucozade", "doesn't taste like piss", "it's the future" and "it's an energy drink with vodka that will put you on your arse"... "DJ constantly made references to alcohol "who is out to get pissed?", "where are all the Glaswegian pissheads?", "who had been drinking all day?" and "people in here who are fucked give me a shout"." (Female Observer Team A, 'Xanadu')

The final function of music in the nightclub experience was in signifying the end of the night (the cessation dancing and bar serving) and controlling the patrons' subsequent exit. Mirroring the increase in tempo (or change in genre, e.g. to happy-hardcore, see earlier field-note) observed earlier in the evening, in the minutes before closing-time some nightclubs were observed gradually winding down the tempo of their music (again indicating the use of set lists, see Hadfield ${ }^{26}$ ). The resultant change in atmosphere could also signify to regulars that the end was nigh, thus helping to promote a more gradual and orderly exit procedure. 
que se acerca el fin de la noche, ayudando a organizar un procedimiento de salida más gradual y ordenado.

"Luces encendidas, pero la gente no empieza a salir hasta la última canción. Pienso que deben poner la misma cada noche, así que cuando suena la gente sabe que es el final". (Observadora femenina, Equipo A, 'Armageddon').

"Al final de la noche se levantaron [personal del bar] y hicieron el baile de Whigfield de 'Saturday night'..." "El DJ anuncia que se dejará de servir bebidas a las 2:50am. Tocan música clásica a partir de las 3:00am." (Observadora femenina, Equipo A, 'Tropicana'- Visita del sábado).

"El personal subió al escenario para bailar 'Cotton-eyed Joe' entonces el DJ dio las buenas noches, se encendieron las luces y tocaron el tema de Rainbow [programa infantil de los años 70]." (Observadora femenina, Equipo A, 'Tropicana' - Visita del viernes).

Como ilustran las dos notas de campo anteriores (del viernes y sábado noche) al describir el procedimiento de descompresión, una vez los locales han dado por terminado su servicio normal de dispensación de bebidas, utilizan una música que difiere ampliamente de aquella en la que el local se especializa, llegando a emplear música clásica y canciones infantiles o, como el siguiente ejemplo ilustra, poniendo viejos éxitos cursis en un local 'hardcore', presumiblemente para evitar que los clientes permanezcan demasiado rato.

"El DJ puso Silver lady de David Soul y itodo el mundo salió, en serio! Esa cosa no se podia aguantar." (Observadora femenina, Equipo $A$, 'Xanadu').

\section{DISCUSIÓN}

Este artículo ha empleado un método de observación de campo para evaluar el papel que juega la música en el manejo de los niveles de comportamiento alterado en los clubes nocturnos. Este método presenta sin embargo algunas limitaciones. Sólo pueden registrarse los fenómenos que han sido observados e, inevitablemente, existe un elemento de subjetividad entre los observadores (aunque esto no siempre juega en detrimento de la investigación). Y, más especificamente, no puede saberse si los operadores o los DJs estaban utilizando intencionadamente la música para conseguir los efectos observados y descritos en el artículo. Sin embargo, esta investigación sustentada sobre un estudio previo realizado en pubs cercanos ${ }^{1}$, que además de utilizar un procedimiento de observación idéntico incorporaba entrevistas cualitativas al personal de los locales (entre los que se encontraban titulares de licencias y monitores de formación de personal de las compañias de cadenas de pubs). El personal de pubs entrevistado indicó que la música se empleaba intencionadamente en las formas observadas (por ejemplo, para incrementar las ventas de bebidas o atraer/eliminar clientes) y como podría esperarse, en el presente estudio sobre los clubes nocturnos, los efectos de la política musical parecen ser mayores que los observados en los pubs.

Se observó que la música influenciaba tanto al tipo de cliente de los clubes como a sus comportamientos una vez dentro, incluyendo los niveles de baile/actividad sexual y los
"Lights up, but people started leaving at last song. I think this may be the same last song every night so when it's played people know it's the end." (Female Observer Team A, 'Armageddon')

"At the end of the night they [bar staff] all got up and did 'Saturday night' Whigfield dance" ... "Last orders called by DJ at 2.50AM. Classical music played after 3.00PM." (Female Observer Team A, 'Tropicana' - Saturday visit)

"Staff got on stage to dance to 'Cotton-eyed Joe' - then DJ said goodnight, lights went up and they played the theme tune to Rainbow [1970s children's TV show]." (Female Observer Team A, 'Tropicana' - Friday visit)

As the two (Friday and Saturday night) field-notes above describing the winding down procedures at 'Tropicana' illustrate, after normal service had ended, during 'drinking-up-time', venues played music which differed greatly to that in which the venue specialised, such as classical, children's songs, or as in the example below an ostensibly very inappropriate cheesy-pop oldie at a 'hardcore' venue, presumably to discourage this nightclub's patrons from lingering too long.

"DJ played Silver lady by David Soul and everyone left, honestly! You couldn't make this stuff up." (Female Observer Team A, 'Xanadu')

\section{DISCUSSION}

This paper utilised a field observation method in order to assess the role that music plays in governing levels of disorderly behaviour within nightclubs. This method has some limitations. Only phenomena actually observed can be recorded, and there will inevitably be an element of subjectivity among observers (though this may not always have been to the research's disadvantage). In particular, it cannot be known whether or not nightclub operators or DJs were intentionally using music for the effects observed and described in this paper. However, this research built upon a previous study conducted in nearby pubs ${ }^{1}$, which as well as using an identical observational procedure, involved qualitative interviews conducted with premises staff (including licensees and a pub chain's company package staff trainer). The pub staff interviewed stated that music was indeed being purposively used in the ways observed (e.g. to increase drinks sales or attract / remove customers) and as might be expected, in the present nightclub study the effects of music policy appeared to be much greater than was observed in the pubs.

Music was observed influencing nightclubs' clientele type and also their behaviours once inside, including dancing / sexual activity and levels of alcohol or other drug use. With the exception of one 'old-skool rave' nightclub, the mainstream homogeneity of the music being played at these 
de consumo de alcohol y otras drogas. Con la única excepción del club 'old-skool rave', la corriente dominante y homogénea de la música empleada en estos locales era asombrosa. Incluso clubes especializados en géneros musicales muy diversos (como happy-hardcore, urbano, o pop-pegadizo) tocaban canciones de artistas de moda (como Kanye West y Pussicat Dolls) o viejos temas (de películas). Esta restringida variedad de artistas/canciones parecería ser utilizada por los operadores de clubes nocturnos como una forma de explotar lo que el psicólogo musical John Davies ${ }^{27}$ denomina el "fenómeno de cariño están tocando nuestra canción" (pág.69) como estrategia de marketing. Si le añadimos el alcohol, esta respuesta emocional provocada por la escucha de una canción familiar puede verse amplificada, quizás alentando mayores gastos en el bar en dicha ocasión en particular, y futuras visitas al mismo club. De hecho, muchos clientes durante el transcurso de esta investigación parecian saber qué locales tocaban el tipo de música que necesitaban, como habia predicho DeNora ${ }^{5}$ (ver Introducción).

De forma similar a lo descubierto por DeNora y Becher en la economía diurna ${ }^{6}$, los operadores parecian saber qué tipo de música necesitaban para manipular los comportamientos de sus clientes, por ejemplo, la utilización de música aparentemente inapropiada al cierre, para desalojar a los mismos clientes a los que se había atraído unas horas antes con otra música. A esto se le denomina 'Método Manilow'28, ${ }^{29}$ y también ha sido utilizado para impedir que los jóvenes estén merodeando alrededor de edificios públicos, centros comerciales, etc. $^{30,32}$ Por ejemplo, una galería de arte de Glasgow (cercana a los clubes nocturnos examinados en este estudio) intentó, sin éxito, evitar que jóvenes góticos merodearan por sus claustros tocando música clásica de forma amplificada ${ }^{33}$. Así que tanto en la economía diurna como en la nocturna la música puede verse creando 'geografías sociales sónicas'.

Estos fenómenos pueden ser vistos como parte de desarrollos conceptuales más amplios, o en debates sobre prevención de delitos a través del diseño ambiental. Estos conceptos no son nuevos, la teoría del 'espacio defendible' ${ }^{34,35}$ de 0 scar Newman, por ejemplo, puede verse aquí en acción, aunque en términos de demarcación de límites sónicos temporales en vez de los físicos fijos. Sin embargo, los hallazgos actuales también encuentran su resonancia con técnicas más recientes desarrolladas por la psicología del comportamiento y los economistas del comportamiento en la 'arquitectura de elección', como las de Sunstein y Thaler ${ }^{36}$ que argumentan que puede darse un ligero 'empellón' ${ }^{37}$ a la gente para que tome decisiones beneficiosas sin necesidad de coerción física o legislativa. En el caso del mantenimiento del orden en los clubes nocturnos, la necesidad de dicho 'empellón' puede ser de gran importancia, dados los efectos de la intoxicación, 0 de la excitación sexual, en la toma de decisiones, y los beneficios obvios de utilizar la 'elección' musical para lograrlo en vez de una 'fuerza' de seguridad.

De acuerdo con estudios previos realizados por el autor en pubs cercanos', la música también fue explorada como factor de riesgo para los desórdenes. Sin embargo, en estos clubes nocturnos, los DJ parecian ser capaces de reaccionar frente a las situaciones y controlar los niveles de orden en venues was striking. In particular, even nightclubs which specialised in very different music genres (e.g. happy-hardcore, urban and cheesy-pop) played songs by the same current artists (e.g. Kanye West and Pussycat Dolls) or oldies (e.g. movie themes). This narrow range of artists / songs would appear to be being used by nightclub operators as a way of tapping into what music psychologist John Davies ${ }^{27}$ has termed the "Darling they're playing our tune phenomenon" (p. $69)$ as a marketing tool. With added alcohol, this emotional response elicited by hearing a familiar song may become amplified, perhaps encouraging increased spending at the bar on that single occasion and future visits to the same nightclub. Indeed many patrons in this research seemed to know which premises were playing the kind of music that they needed as predicted by DeNora ${ }^{5}$ (see Introduction).

Similarly as found DeNora and Belcher in the Day-time Economy ${ }^{6}$, operators seemed to know what kind of music they needed to manipulate their customers' behaviour, for example the use of apparently inappropriate music at closing-time to remove the very patrons who only hours before they had used music to attract. This has been termed the 'Manilow Method'28, 29 and it has been also used to deter young people from loitering around public buildings, shopping centres etc. ${ }^{30-32}$ For example, a Glasgow art gallery (near the nightclubs observed in this research) attempted, unsuccessfully, to deter Goth youths from their cloisters by playing amplified classical music ${ }^{33}$. Thus in both the Day-time ${ }^{6}$ and Night-time Economies music can be seen creating a 'sonic social geography'.

Such phenomena can be viewed as part of wider conceptual developments and debates in crime prevention through environmental design. Such concepts are not new, for example Oscar Newman's theory of 'defensible space' ${ }^{14,35}$ can be seen in operation here, albeit in terms of demarking temporal sonic boundaries rather than fixed physical ones. However the present findings also resonate with more recent techniques being developed in behavioural psychology and by behavioural economists into 'choice architecture', such as Sunstein and Thaler ${ }^{36}$ who argue that people can be given a gentle 'nudge'37 into making beneficial decisions without the need for physical or legislative coercion. In the case of maintaining good order within nightclubs the need for a 'nudge' may be of particular importance because of the effects of intoxication, or arousal, upon decision making, and the obvious benefits of using music 'choice' for this end rather than a security 'force'.

In common with previous research conducted by the author in nearby pubs ${ }^{1}$, music was again observed to be a risk factor for disorder. However in these nightclubs, DJs appeared to be able to react to situations and control levels of order to a much greater extent than was the case in the pubs. In this way music may be viewed a form of 'soft policing' (as opposed to the 'hard policing' of security staff, door policies, CCTV, metal detectors and the ultimately calling the 
un mayor grado que en el caso de los pubs. De esta forma, la música puede ser vista como una forma de 'vigilancia blanda' (en oposición a la 'vigilancia dura' del personal de seguridad y de puerta, los circuitos cerrados de televisión, los detectores de metal y, en última instancia, la llamada a la policia) dentro de la economía nocturna, y a los DJs como 'controladores blandos'20.

En su evaluación sobre el riesgo de violencia en los locales de copas australianos, Homel y Tomsen anotaron que podia emplearse la música para esta 'vigilancia blanda'. En aquel estudio concluyeron que "una pequeña multitud con una mala banda parecía más propensa a presentar problemas que una gran multitud entretenida por músicos de calidad". Sin embargo, el presente estudio formula objeciones a términos tan ambiguos como "mala" o "calidad". Los músicos de calidad (es decir, cualificados), o en el caso actual los DJ de buena calidad, también tienen el poder de hacer "mal" las cosas, ya sea promocionando irresponsablemente el alcohol, fomentando atmósferas intimidantes, atrayendo a una multitud problemática o incluso incitando a la violencia. Esto queda ilustrado en el siguiente relato, tomado de una investigación llevada a cabo por James C. Roberts en los EE.UU. (1997, citada por Graham y Homel ${ }^{4}$, pág. 123).

"Nosotros, definitivamente, podemos contribuir en la violencia en el bar. Por ejemplo, hace unos años, cuando esto bares permitían que se hiciera "moshing" [bailar saltando y dando empujones], solia agrupar canciones de forma que sabía que incitarian a la pelea y a los incidentes con agresiones. Ahora, tengo que poner un par de canciones suaves entre las más duras para que la gente no se descontrole... Hace un par de semanas... Miré a uno de los chicos de la banda y le dije, "Mira esto, voy a hacer que esta gente se desmadre". Tuvimos que dejar de tocar durante diez minutos mientras los gorilas del local intentaban sacar a los que se peleaban fuera del bar. Habian lanzado a un chico sobre la mesa de sonido y no pudimos volver a enchufar los instrumentos. Fue un completo desastre". (Cantante solista, Hombre, 'Banda X').

El presente estudio también identificó el fenómeno descrito más arriba (aunque las intenciones de los DJs, que de forma repentina cambiaban la atmósfera, eran desconocidas). Un fenómeno que no puede ser visto como un reflejo de la 'calidad' artística o técnica de la música implicada. Quizás lo más preocupante aqui seria la observación de que los DJs estaban siendo utilizados para promocionar abiertamente marcas de bebidas alcohólicas y de hecho borracheras (ver también, Home Office / KPMG LLP ${ }^{38}$; Turney ${ }^{39}$ ).

De este modo, este estudio pone en evidencia los límites de esta investigación, que deberia garantizar próximos estudios, mediante otros métodos (como las entrevistas) que incluyeran temas como de qué forma la música afecta las ventas en los bares, la interacción entre la música y las sustancias de consumo, o la música y los comportamientos sexuales, y especialmente de qué forma ven estos animadores su papel en la economía nocturna. Las futuras investigaciones deberían centrarse en el punto de vista de los clientes y del personal trabajando en la economía nocturna $y$, en particular, en las experiencias de músicos y DJs. Por ejemplo, hasta qué punto los operadores de clubes nocturnos (o pubs) emplean a los DJs como focos de atracción, como contro- police) within the Night-time Economy, and DJs as 'soft controllers'20.

In their assessment of Australian drinking venues' violence risk, Homel and Tomsen ${ }^{19}$ also noted that music could be used for such 'soft policing'. At that time they concluded that "A smaller crowd with a bad band seems more likely to present trouble than a large crowd entertained by quality musicians." However, the present research does raise questions over such ambiguous terms as "bad" or "quality". Quality (i.e. skilled) musicians, or in the present case good quality DJs, will have it within their power to do 'bad' things, whether that be irresponsible alcohol marketing, fostering an intimidating atmosphere, attracting a troublesome crowd or even inciting violence. This is illustrated by the account below, which is taken from research conducted by James C. Roberts in the USA (1997 quoted by Graham and Homel ${ }^{4}$, p. 123).

"We could definitely contribute to violence in the bar. For example, years ago, when many of these bars would allow moshing to occur, I used to group songs together that I knew would incite fights and incidents of aggression. Now, I have to play a couple of mellow songs between heavier songs so that people don't get so riled up ... Two weeks ago... I turned to one of the guys in the band and said, "Watch this, I'm going to bring these people over the edge". We had to stop playing for ten minutes while the bouncing staff tried to clear people who were fighting out of the bar. One guy got thrown into the sound board and we couldn't get the instruments turned back on. It was a real mess." (Male lead singer, 'Band $X$ ').

The present research also identified the above phenomenon (though the intentions of the DJs who suddenly changed the atmosphere was not known), one which cannot be viewed as a reflection of the artistic or technical 'quality' of the music concerned. Perhaps of more concern here however, was the observation that DJs were being employed to overtly promote alcoholic drinks brands and indeed drunkenness (see also Home Office ( KPMG LLP ${ }^{38}$; Turney ${ }^{39}$ ).

Thus the present study has raised a number of new research frontiers which warrant further investigation by other methods (e.g. interviews), including how music affects bar sales, the interaction between music and substance use, or music and sexual behaviours, and importantly how entertainers view their role in the Night-time Economy. Future research should focus the views of patrons and staff working in the Night-time Economy, in particular the experiences of musicians and DJs. For example, to what extent do nightclub (or pub) operators employ DJs as an attractor, as crowd controllers or as drinks promoters? How much artistic freedom are such DJs given (e.g. are they de-skilled to playing a set-list pre-arranged by operators, and if so is this for reasons of marketing or public order)? How do DJs manage their set list (if they are allowed to) and how do they balance the competing demands of economics, public safety and artistic concerns? 
ladores de multitudes o como promotores de bebidas. Qué libertad artística se les da a dichos DJs (es decir, se prescinde de sus habilidades al dárseles una lista pre-elaborada por el operador, y si es así ¿se hace por razones de marketing o de orden público?), cómo gestionan los DJs su lista fija (en caso que se les permita hacerlo) y como equilibran la competencia entre las demandas económicas, las de seguridad pública y las inquietudes artísticas.

Entretanto, las implicaciones del estudio actual son claras. La música debería ser manejada con cuidado por parte de los operadores de clubes nocturnos y DJs. Por ejemplo, la política musical debería estar integrada en el diseño de los establecimientos, para evitar congestiones en la zona de baile y permitir acústicas diversas, de tal forma que los clientes pudieran conversar y pedir bebidas ( $\sin$ sentirse frustrados) y que el personal de seguridad pudiera oír los problemas (o se les pudiera indicar dónde ocurren). Hace falta reconocer el papel que juega la música en el marketing de bebidas $y$, en oposición a esto, en la minimización de políticas de control del desorden. Para ello, los programas de dispensación responsable de bebidas (DRB) y las formaciones en minimización de desórdenes deberían hacerse extensibles a los animadores, especialmente a los DJs. Sin embargo, esto no debería hacerse a expensas de limitar el acceso a cualquier género musical considerado 'problemático' (en este estudio, tan sólo la 'old-skool rave' pasó el examen sin fomentar comportamientos desordenados).

En resumen, la música es más que entretenimiento, constituye una forma de control de la multitud / mente. Resulta sorprendente que el poder de la música, para establecer el orden dentro de la economía nocturna, haya sido pasado por alto por tantos investigadores académicos, lo que Hadfiel ${ }^{40}$ ha llamado el 'gobierno sónico'. ¿Por qué, por ejemplo, deberian los militares mostrarse más interesados en la música, ya sea marchando al ritmo del tambor o al presunto uso de temas infantiles de TV en la bahía de Guantánamo ${ }^{32,41-43}$ que, digamos, al sexo o las drogas? Volviendo a lan Dury, 'el rock $n$ ' roll' ha jugado el papel de 'Cenicienta', frente al sexo o las drogas, en el campo del Club Health. Desafortunadamente, las cuestiones evidenciadas en este estudio vienen de antiguo, como ilustra el siguiente relato de un periódico, sobre un desorden que tuvo lugar hace 90 años en un local (que sigue en activo en el momento de escritura del artículo), en la misma ciudad donde el trabajo de campo para este estudio se ha llevado a cabo.

"[la víctima] la mujer de un soldado ahora en servicio activo, dijo que estaba en un salón de baile en la carretera de North Woodside [Glasgow] durante la noche del último 23 de octubre. A la acusada le pareció mal que ella cantara mientras sonaba la música. Más tarde, en una sala anexa, le golpeó la cara y estalló la pelea..." (Scotsman, 8 de noviembre de 1916).

\section{CONCLUSIÓN}

Esta investigación ha puesto de manifiesto de qué forma la música constituye una parte integral de la salud en los clubes. Aunque la música puede avivar una agradable salida
Meantime the implications of the current research are clear. Music should be handled with care by nightclub operators and DJs. For example, music policy should be integral to premises design, to avoid dancing congestion and to allow for variable acoustics so that patrons are able to converse and order drinks (without becoming frustrated) and so security staff can hear trouble (or be told where the trouble is located). There is a need to recognize the role of music in drinks marketing and (perhaps opposing) disorder minimisation policies. As such Responsible Beverage / Bar Service (RBS) programs and disorder minimisation training should be made available to entertainers, especially DJs. However, this should not be at the expense of limiting access to any musical genres deemed 'problematic' (in this study, only 'old skool rave' was not observed fostering some disorderly behaviour).

In summary, music is more than just entertainment it is a form of crowd / mind control. It is surprising that the power of music to establish order within the Night-time Economy has been largely overlooked by academic researchers, what Hadfield ${ }^{40}$ has termed 'sonic governance'. Why for example should the military be more interested in music, as opposed to say sex or drugs, whether from marching to the beat of the first drum to the alleged playing of children's TV themes at Guantanamo Bay ${ }^{32,41-43}$ ? Returning to Ian Dury, 'rock n' roll' has played 'Cinderella' to sex and drugs for too long in the field of Club Health. This is unfortunate as the issues raised by this study are longstanding, as is illustrated by the following newspaper account of a disorderly incident which took place 90 years previously in a venue (which was still trading at the time of writing) in the same city as where the fieldwork for the present research took place.

"[the victim] was the wife of a soldier now on active service, said she was in a dancing hall in North Woodside Road [Glasgow] on the night of $23^{\text {rd }}$ October last. Accused found fault with her singing during the music. Later in a side room, she struck her in the face and there was a fight..." (Scotsman, November $8^{\text {th }} 1916$ )

\section{CONCLUSION}

This research has highlighted how music is an integral part of club health. Although music can foster an enjoyable night out, as was observed during this research, it can also have a dark-side, one which if not properly managed can result in problems. A venue's music policy can act as a receptor of disorder, through the clientele which it may attract, and also as a generator of disorder, through encouraging their rowdy behaviour. However it can also be used to discourage disorder and it is concluded that advice on music policy should become an essential component of nightlife violence reduction strategies and that appropriate training be made available to both entertainers and nightclub operators. 
nocturna, como se ha observado durante este estudio, si no se gestiona debidamente puede constituir un problema. La política musical de un local puede actuar como un receptor del desorden, a través de la clientela que atrae, y también como un generador de desórdenes, a través del fomento de sus comportamientos alborotados. Sin embargo, también puede ser empleada para poner freno al desorden. $Y$, para concluir, un asesoramiento sobre la política musical debería ser un componente esencial en las estrategias de reducción de violencia de la vida nocturna y debería proveerse una formación adecuada tanto a los animadores como a los operadores de clubes nocturnos.

\section{AGRADECIMIENTOS:}

El autor quisiera expresar su agradecimiento a Andy Boyd, Jemma Lennox, Katie 0'Neil y Tom Woods que actuaron como fieles observadores durante el desarrollo de esta investigación. Este artículo está financiado por el Alcohol Education Research Council (AERC) y se presentó en la sesión paralela $1 \mathrm{C}$ de la Conferencia Club Health celebrada en Santa Eulàlia des Riu, Ibiza, el 24 de junio de 2008.

\section{REFERENCIAS / REFERENCES}

1. Forsyth AJM, Cloonan M. Alco-pop?: The Use of Popular Music in Glasgow Pubs. Pop Music Soc 2008;31:57-78.

2. Brosnahan A. Like it Loud: Don't lose the music campaign from improved hearing health in clubs. Paper presented at Club Health 2008; 2008 Jun 24; Santa Santa Eulària des Riu, Eivissa (lbiza), Spain.

3. McDonald L. How to avoid a Noise Hangover: Sound advice for clubbers and clubs. Paper presented at Club Health 2006, 2006; Sep 23; Gea College, Piran, Slovenia.

4. Graham K, Homel R. Raising The Bar: Preventing violence in and around bars, pubs and clubs. Devon: Willan; 2008.

5. DeNora T. Music in Everyday Life. Cambridge: Cambridge University Press; 2000.

6. DeNora T, Belcher S. 'When you're trying something on you picture yourself in a place where they are playing this kind of music': Musically Sponsored Agency in the British Retail Clothing Sector. Sociol Rev 2000;48:80-101.

7. DeNora T. Music as a technology of the self. Poetics 1999;27:31-56.

8. Fegaon $S$, Carmichael C. Why heavy metal and classical fans make sweet music together. The Herald 2008 Sep 4 [cited 2009 Sep 24]; Available from: URL:http://www.theherald.co.uk/news/ news/display.var.2441316.0.Why_heavy_metal_and_classical_ fans_make_sweet_music_together.php

9. Michaels S. Classical and metal fans: birds of a feather? The Guardian 2008 Sep 4 [cited 2009 Sep 24]; Available from: URL:http://www.guardian.co.uk/music/2008/sep/08/classical. metal.fans.study

10. North A, Hargreaves D. Music and consumer behaviour. In: Hargreaves D, North A, editors. The Social Psychology of Music. Oxford: Oxford University Press; 1997. p. 268-82.

\section{ACKNOWLEDGEMENTS:}

The author would like to thank Andy Boyd, Jemma Lennox, Katie 0'Neil and Tom Woods who acted as the field observers in this research.

This paper is based on research funded by the Alcohol Education Research Council (AERC) and presented in Parallel Session 1c at the Club Health 2008 conference held at Santa Eulària des Riu, Eivissa, 24 $4^{\text {th }}$ of June 2008.

11. Areni CS, Kim D. The influence of background music on shopping behaviour - classical versus top-40 music in a wine store. Adv Consum Res 1993;20:336-40.

12. Center on Alcohol Marketing and Youth. Radio Daze: Alcohol Ads Tune in Underage Youth. Washington DC: Center on Alcohol Marketing and Youth, Georgetown University; 2003.

13. Daykin $\mathrm{N}$, Orme J. The role of alcohol in weekend radio output aimed at young people in England: A mixed Methods study. Paper presented at the British Sociological Association MedSoc conference; 2008 Sep 5; Brighton England, UK.

14. Drews DR, Vaughn DB, Anfiteatro A. Beer consumption as a function of music and the presence of others. J Pennsylvania Acad Sci 1992;65:134-36.

15. Guéguen N, Jacob C, LeGuellec $H$, Morineau T, Lourel M. Sound Level of Environmental Music and Drinking Behavior: A field experiment with beer drinkers. Alcohol Clin Exp Res 2008;32:1795-98.

16. McElrea H, Standing L. (1992) Fast music causes fast drinking. Percept Mot Skills 1992;75:362.

17. Bach PJ, Schaefer JM. The tempo of country music and the rate of drinking in bars. J Stud Alcohol 1979:40;1058-59.

18. Caldwell $C_{1}$ Hibbert SA. Play that one again: The effect of music tempo on consumer behaviour in a restaurant. In: Dubois $B_{1}$ Lowrey TM, Shrum $\sqcup$, Vanhuele M, editors. European Advances in Consumer Research Volume 4. Provo, UT: Association for Consumer Research; 1999. p. 58-62.

19. Homel R, Tomsen S. Hot Spots for Violence: The environment of pubs and clubs. In: Strang H, Gerull S-A, editors. Homicide: Patterns, Prevention and Control Canberra. Canberra: Australian Institute of Criminology; 1993. p. 53-66.

20. Hadfield P. Bar Wars: Contesting the night in contemporary British cities. Oxford: Oxford University Press; 2006

21. Brewster B, Broughton F. Last Night a DJ Saved My Life: The history of the disc jockey. London: Hodder Headline; 2006. 
22. Graham K. Safer Bars: Assessing and Reducing Risks of Violence. Toronto: Centre for Addiction and Mental Health; 1999.

23. Graham K. Safer Bars: Training Manual for Observers on the Safer Bars Study. Toronto: Centre for Addiction and Mental Health; 2000.

24. Purcell J, Graham K. A typology of Toronto Nightclubs at the turn of the Millennium. Contemp Drug Probl 1995;32;131-67.

25. Portman Group. Code of Practice: On the naming, packing and promotion of alcoholic drinks, $3^{\text {rd }}$ Edition. London: Portman Group; 2002.

26. Hadfield P. The Prevention of Public Disorder. In: Kolvin P, editor. Licensed Premises: Law and Practice. London: Tottel; 2004:567-617.

27. Davies JB. The Psychology of Music. London: Hutchinson; 1978.

28. Hirsch LE. Weaponizing Classical Music: Crime prevention and symbolic power in the age of repetition. J Pop Music Stud 2007;19:342-58

29. Editorial. Cruel and Unusual Manilow. 2008 Sep 4 [cited 2009 Sep 24]; Available from: URL:http://www.rollingstone. com/blogs/smokingsection/2008/11/guilty-you-are-nowsentenced-t.php

30. Ananova. Cliff helps clear pubs. 2004 [cited 2009 Sep 24]; Available from: URL:http://www.ananova.com/news/story/ sm_1205258.html

31. BBC News. Music to deter Yobs by. 2005 Jan 10 [cited 2009 Sep 24]; Available from: URL:http://news.bbc.co.uk/1/hi/ magazine/4154711.stm

32. Cloonan M, Johnson B. Killing me softly with his song: An initial investigation into the use of popular music as a tool of oppression, Pop Music 2002;21;7-39.
33. Horne M. Goma Goths Banned - Heaven knows they're miserable now. The Scotsman 2007 Dec 9 [cited 2009 Sep 24]; Available from: URL:http://www.rickross.com/reference/goth/goth13.html

34. Newman 0. Defensible Space: Crime prevention through urban design. New York: Macmillan; 1972.

35. Newman 0. Creating Defensible Space. Washington: US Department of Housing and Urban Development (HUD); 1996 [cited 2009 Sep 24]; Available from: URL:http://www.huduser.org/ publications/pdf/def.pdf

36. Sunstein $\mathrm{CR}$, Thaler RH. Libertarian Paternalism is not an Oxymoron. Univ Chic Law Rev 2003;70:1159-202.

37. Thaler RH, Sunstein CR. Nudge: Improving decisions about health wealth and happiness. New York: Penguin; 2008.

38. Home Office / KPMG LLP. Review of the Social Responsibility Standards for the Production and Sale of Alcoholic Drinks. Birmingham: KPMG LLP; 2008.

39. Turney E On-trade 'guilty of irresponsible practices'. Morning Advertiser: Officially the pub trade's favourite; $2008 \mathrm{Jul} 21$.

40. Hadfield P. Nightlife Horizons: Some further thoughts and conclusions. In: Hadfield P, editor. Nightlife and Crime: Social Order and Governance in International Perspective. Oxford: Oxford University Press; 2009; 331-54

41. Bayoumi M. Disco Inferno. The Nation, 2005;32-5 Dec 26 [cited 2009 Sep 24]; Available from: URL:http://www.thenation.com/ doc/20051226/bayoumi

42. Cusick SG. Music as torture / Music as weapon. Transcult Music Rev 2006;10 [cited 2009 Sep 24]; Available from: URL:http:// www.sibetrans.com/trans/trans10/cusick_eng.htm

43. Johnson B, Cloonan M. Dark Side of the Tune: Popular music and violence. Aldershot: Ashgate; 2008 
